\title{
Age-dependent neurodegeneration and organelle transport deficiencies in mutant TDP43 patient-derived neurons are independent of TDP43 aggregation
}

\author{
Kreiter $\mathbf{N}^{1,2}, \underset{3}{\operatorname{Pal}} \mathbf{A}^{1}, \operatorname{Lojewski} \mathbf{X}^{1}, \operatorname{Corcia} \mathbf{P}^{4}, \underset{1,2,3,6}{\operatorname{Naujock}} \mathbf{M}^{5+}, \underset{1,2,3^{*}}{\operatorname{Reinhardt}} \mathbf{P}^{3 \#}$, \\ Sterneckert J , Petri, $S^{5}$, Wegner $F^{5}$, Storch A and Hermann A \\ ${ }^{1}$ Department of Neurology, Technische Universität Dresden, Germany \\ ${ }^{2}$ German Center for Neurodegenerative Diseases (DZNE), Dresden, Germany \\ ${ }^{3}$ Center for Regenerative Therapies Dresden (CRTD), Technische Universität Dresden, Germany \\ ${ }^{4}$ Centre expert pour la SLA et les maladies du motoneurone CHU Tours 2 Boulevard TONNELLE 37044 \\ Tours CEDEXI \\ ${ }^{5}$ Department of Neurology, Hannover Medical School, Hannover, Germany \\ ${ }^{6}$ Department of Neurology, University of Rostock, Rostock, Germany \\ " current address: AbbVie Germany GmbH \& Co KG, Neuroscience Discovery, Ludwigshafen am Rhein, \\ Germany \\ ${ }^{+}$current address: Boehringer Ingelheim Pharma GmbH \& Co. KG, CNS Research Department, Germany
}

running title: TDP43-proteinopathy is independent of protein aggregation

\section{* Corresponding author:}

Prof. Dr. med. Dr. rer. med. Andreas Hermann

Department of Neurology

Technische Universität Dresden

Fetscherstraße 74, 01307 Dresden, Germany

Phone: ++49-351-458-2532; Fax: ++49-351-458-4352

e-mail: Andreas.Hermann@uniklinikum-dresden.de 


\begin{abstract}
TAR DNA-binding protein 43 (TDP43) is a cause of familiar and sporadic amyotrophic lateral sclerosis (ALS). The diverse postulated mechanisms by which TDP43 mutations cause the disease are not fully understood. Human wildtype and TDP43 S393L and G294V mutant spinal motor neuron cultures were differentiated from patient-derived iPSCs. Mutant hTDP43 and wildtype motor neuron cultures did not differ in neuron differentiation capacity during early maturation stage. During aging we detected a dramatic neurodegeneration including neuron loss and pathological neurofilament abnormalities in TDP43 mutant cultures only. Additionally mitochondria and lysosomes of aging spinal motor neurons revealed robust TDP43 mutation dependent abnormal phenotypes in size, shape, speed and motility which all appeared without TDP43 mislocalization or aggregation formation. Furthermore, D-sorbitol - known to induce stress granules and cytoplasmic mislocalization of TDP43 - rescued axonal trafficking phenotypes without any signs of TDP43 mislocalization or aggregation formation. Our data indicate TDP43 mutation-dependent but cytosolic aggregation-independent mechanisms of motor neuron degeneration in TDP43 ALS.
\end{abstract}

Keywords: Amyotrophic lateral sclerosis, TAR DNA binding protein 43, TARDBP, neurodegeneration, mitochondria, lysosomes, live cell imaging, axonopathy 


\section{Introduction}

Worldwide 2-4 of 100.000 people per year suffer from amyotrophic lateral sclerosis (ALS) which is the most frequent variant of motor neuron disease with a survival time of 1-5 years after symptom onset. Motor neurons are the mainly affected cell type that undergoes degeneration and death in ALS ${ }^{1,2}$. Several gene mutations were found to cause familiar forms of ALS (fALS) of which the first identified were SOD1 (superoxide dismutase 1) gene mutations in $1993^{3,4}$. In 2006 mutations in TDP43 (TAR DNA binding protein, TDP43) were identified to cause fALS as well as sporadic ALS but the pathophysiology caused by mutant TDP43 is still not fully understood ${ }^{5}$. Most importantly, TDP43 is the main aggregating protein in sporadic ALS also being aggregated in some fALS forms (e.g. TDP43, C9ORF72) ${ }^{6}$. However, TDP43-mediated pathophysiology appears to be mechanistically distinct from mutant SOD1 action ${ }^{7}$.

Different mechanisms for TDP43 mutant pathology have been suggested (for review see Jovicic). These include nuclear loss of function leading to transcription and splicing defects, toxic properties in the cytoplasm sequestering RNAs and RNA binding proteins and finally loss of axonal transport function with RNA granule transport deficiencies ${ }^{8}$. The main focus has been drawn on pathological accumulation of cytosolic TDP43 ${ }^{9-11}$. Mutant TDP43 human induced pluripotent stem cell (hiPSC)-derived neurons were reported to show elevated levels of soluble and detergent- resistant TDP43 protein and decreased survival in long-term differentiation ${ }^{12}$. Such lines showed a two-fold increase in cytosolic TDP43 compared to the controls which could be - at least partially - reduced by $30 \%$ by allele-specific knockdown ${ }^{13}$. However, on a closer look, also in models reporting cytosolic mislocalization of TDP43, only up to one third of cells showed this phenotype while the remaining cells still showed physiological nuclear TDP43 $10,11,14$.

TDP43 was shown to be actively transported within the axon in both directions ${ }^{14}$. TDP43 thereby forms cytoplasmic mRNP granules that undergo bidirectional, microtubule-dependent transport in neurons and facilitate delivery of target mRNA to distal neuronal compartments ${ }^{15}$. TDP43 mutations interfere with mRNA transport function ${ }^{14,15}$. Neurofilament light chain (NEFL) mRNA was shown to be transported in such TDP43 granules and was reduced in TDP43 mutant motor neurons ${ }^{10,15}$.

ALS-linked mutations in TDP43 can impair stress granule dynamics ${ }^{16}$. TDP43 had previously been shown to associate with multiple proteins that are part of mRNP granules (e.g., staufen, FMRP, SMN, and HuD), including neuronal transport granules ${ }^{17}$. TDP43 was shown to be directed to stress granules by sorbitol, a novel physiological osmotic and oxidative stressor ${ }^{18}$ and to regulate FOXO-dependent protein quality control in stress response ${ }^{19}$. 
Mutations in TDP43 led to abnormal ${ }^{9}$ or reduced neurite outgrowth ${ }^{20,21}$. TDP43 mice model shows lack of mitochondria in motor axon terminals ${ }^{10}$. Furthermore, TDP43 was reported to impair mitochondria and lysosome morphology and function in cell models of ALS. Interestingly, both overexpression and knockdown caused perturbations in primary murine neurons suggesting a tight regulation of TDP43 ${ }^{22,23}$. Overexpression of wildtype or mutant TDP43 caused mitochondrial shortening in dendrites, not in axons, due to increased fission and decreased mitochondrial movement in axons and dendrites. In contrast, knockdown increased mitochondrial length but also decreased dendritic and axonal mitochondrial motility. Mt TDP43 caused co-staining of mitochondria and TDP43, which was rescued by Mfn2 expression.

However, the relation between (stress induced) cytoplasmic TDP43 aggregation and neuronal dysfunction and degeneration remains enigmatic. Furthermore, data on organelle trafficking and neurite morphology in human patient-derived motor neurons are still lacking.

This prompted us to use patient-derived induced pluripotent stem cells (hiPSCs)-derived motor neurons to investigate (i) the sequential appearance of neuronal dysfunction and neurodegeneration, (ii) aggregate formation and (iii) how these events are mechanistically connected. Surprisingly, we found significant neuronal dysfunction and degeneration during cellular aging, but did not find relevant signs of pathological TDP43 aggregation, arguing against an upstream function of TDP43 aggregation in TDP43-ALS. 


\section{Materials and Methods}

\section{Patient characteristics}

We included cell lines carrying a "benign" (S393L, late onset primary anarthria with ALS/LMND, no clinical symptoms of FTD, female, family history of ALS and PD) and a “malign” (G294V, early onset ALS, no clinical symptoms of FTD, male, no family history) TDP43 mutation and were compared to four wildtype cell lines from healthy volunteers (female, age at biopsy 45 ; female, age at biopsy 53; male, age at biopsy $60^{24,25}$ ). An overview of the used cell lines is demonstrated in Supplemental Table S1. The performed procedures were in accordance with the Helsinki convention and approved by the Ethical Committee of the University of Dresden (EK45022009; EK393122012). Informed consent was obtained from any individual including informed consent about publishing data obtained from the iPSCs derived from skin fibroblasts. The skin biopsies (see below) were obtained after anonymisation.

\section{Generation of iPSC lines}

For generating iPSC lines fibroblast lines were established from skin biopsies taken from familial ALS patients and healthy controls. The generation and characterization of wildtype iPSC lines was reported previously ${ }^{24,26-28}$. Briefly, patient fibroblasts were reprogrammed using pMXbased retroviral vectors encoding the human cDNAs of OCT4, SOX2, KLF4 and cMYC (pMX vectors). Vectors were co-transfected with packaging-defective helper plasmids into 293T cells using Fugene 6 transfection reagent (Roche Diagnostics). Fibroblasts were plated at a density of 50,000 cells/well on $0.1 \%$ gelatin-coated 6-well plates and infected three times with a viral cocktail containing vectors expressing OCT4:SOX2:KLF4:cMYC in a 2:1:1:1 ratio in presence of $6 \mu \mathrm{g} / \mathrm{ml}$ protamine sulfate (Sigma Aldrich) and $5 \mathrm{ng} / \mathrm{ml} \mathrm{FGF2} \mathrm{(Peprotech).} \mathrm{Infected}$ fibroblasts were plated onto mitomycin $\mathrm{C}$ (MMC, Tocris) inactivated $\mathrm{CF}-1$ mouse embryonic fibroblasts (in-lab preparation) at a density of 900 cells/cm 2 in fibroblast media. The next day media was exchanged to ES medium containing 78\% Knock-out DMEM, 20\% Knock-out serum

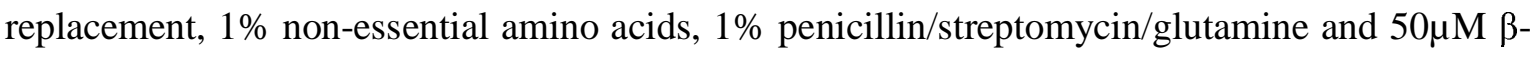
Mercaptoethanol (all from Invitrogen) supplemented with $5 \mathrm{ng} / \mathrm{ml}$ FGF2 and $1 \mathrm{mM}$ valporic acid (Sigma Aldrich). Media was changed every day to the same conditions. iPSC-like clusters started to appear at day 7 post infection, were manually picked 14 days post-infection and plated onto CF-1 feeder cells in regular ES-Media containing $5 \mathrm{ng} / \mathrm{ml} \mathrm{FGF2.} \mathrm{Stable} \mathrm{clones} \mathrm{were} \mathrm{routinely}$ passaged onto MMC-treated CF-1 feeder cells (Globalstem) using $1 \mathrm{mg} / \mathrm{ml}$ collagenase type IV (Invitrogen) and addition of $10 \mu \mathrm{M}$ Y-27632 (Ascent Scientific) for the first 48 hours after passaging. Media change with addition of fresh FGF2 was performed every day. 
Stable clones were analyzed by qRT-PCR for silencing of viral transgenes prior to further experimental procedures.

\section{Trilineage differentiation potential}

iPSC colonies were grown under standard conditions, cleaned and treated with collagenase type IV (2mg/ml, Invitrogen). Floating aggregates were collected and transferred into ultra-low attachment plates (NUNC) in regular ES-Media containing $5 \mu \mathrm{M}$ Y-27632 (Ascent Scientific) for meso-/endodermal differentiation or ES-Media containing $5 \mu \mathrm{M}$ Y-27632, $10 \mu \mathrm{M}$ SB431542 (Tocris) and $1 \mu \mathrm{M}$ Dorsomorphin (Tocris) for ectodermal differentiation. Two days later the medium was changed to the same conditions leaving out the Y-27632. After four days of EB formation, aggregates were plated onto gelatin $(0.1 \%$, Millipore) coated wells for meso/endodermal differentiation or onto plates coated with MatrigelTM (BD Bioscience) for ectodermal differentiation. EBs were differentiated for two weeks using 77.9\% DMEM (high glucose, Invitrogen), 20\% FCS (PAA), 1\% non-essential amino acids (Invitrogen), 1\% penicillin/streptomycin/glutamine (Invitrogen) and 0.1\% $\beta$-Mercaptoethanol (Invitrogen) for the meso-/endodermal lineage and 50\% DMEM/F12 (Invitrogen), 50\% Neurobasal (Invitrogen) containing 1:200 N2 supplement (Invitrogen), 1:100 B27 supplement without vitamin A (Invitrogen), $1 \%$ penicillin/streptomycin/glutamine, 0.1\% $\beta$-Mercaptoethanol and 1:500 BSA Fraction V (Invitrogen) for ectodermal differentiation.

\section{Karyotyping}

TDP43 iPSC and wildtype cell lines were karyotyped using the HumanCytoSNP-12v array. All clones showing pathological SNPs were excluded (data not shown).

\section{Genotyping}

TDP43 iPSC lines were genotyped after all other characterization had been finished. This was done by a diagnostic human genetic laboratory (CEGAT, Tübingen, Germany) using diagnostic standards.

\section{Differentiation of human NPCs to spinal motor neurons}

The generation of human NPCs and motor neurons was accomplished following the protocol from Reinhardt et al. ${ }^{26}$. In brief colonies of iPSCs were collected and stem cell medium was added containing 10 $\mu \mathrm{M}$ SB-431542, $1 \mu \mathrm{M}$ Dorsomorphin, $3 \mu \mathrm{M}$ CHIR 99021 and $0.5 \mu \mathrm{M}$ purmorphamine ${ }^{29}$. After 2 days hESC medium was replaced with N2B27 consisting of the aforementioned factors and DMEM-F12/ Neurobasal 50:50 with 1:200 N2 Supplement, 1:100 B27 lacking Vitamin A and 1\% penicillin/ streptomycin/glutamine. On day 4 150 $\mu \mathrm{M}$ Ascorbic 
Acid was added while Dorsomorphin and SB-431542 were withdrawn. 2 Days later the EBs were mechanically separated and replated on Matrigel coated dishes. For this purpose Matrigel was diluted 1:100 in DMEM-F12 and kept on the dishes over night at room temperature. Possessing a ventralized and caudalized character the arising so called small molecule NPCs (smNPC) formed homogenous colonies during the course of further cultivation. It was necessary to split them at a ratio of 1:10 - 1:20 once a week using Accutase for $10 \mathrm{~min}$ at $37^{\circ} \mathrm{C}$.

Final motor neuron differentiation was induced by treatment with $1 \mu \mathrm{M}$ PMA in N2B27 exclusively. After 2 days $1 \mu \mathrm{M}$ retinoic acid (RA) was added. On day 9 another split step was performed to seed them on a desired cell culture system. Furthermore the medium was modified to induce neuronal maturation. For this purpose the developing neurons were treated with N2B27 containing $10 \mathrm{ng} / \mu \mathrm{l} \mathrm{BDNF}, 500 \mu \mathrm{M}$ dbcAMP and $10 \mathrm{ng} / \mu \mathrm{l} \mathrm{GDNF}$. Following this protocol it was possible to keep the cells in culture for over 2 months. The cells were analyzed at day 5 and day 32 post maturation induction by immunofluorescence imaging.

\section{Immunofluorescence of spinal motor neurons}

For immunofluorescence staining, cells were washed twice with PBS without $\mathrm{Ca} 2^{+} / \mathrm{Mg} 2^{+}$ (LifeTechnologies) and fixed with 4\% PFA in PBS for 15 min at RT. PFA was aspirated and cells were washed three times with PBS. Fixed cells were first permeabilized for 10 minutes in $0.2 \%$ Triton X solution and subsequently incubated for 1 hour at RT in blocking solution (5\% donkey serum in PBS). Following blocking, primary antibodies were diluted in PBS and cells were incubated with primary antibody solution overnight at $4^{\circ} \mathrm{C}$. The following primary antibodies were used: chicken anti-SMI32 (1:10000, Covance), rabbit anti-beta-III-Tubulin (1:3000, Covance), rabbit anti-CHAT (1:1500, Chemicon), mouse anti-MAP2 (1:500, BD Biosciences). Post to the primary antibodies cells were washed three times for 5 min with PBS. Secondary antibodies were diluted in PBS and incubated with the cells for 1.5 hours at RT. Following secondary antibodies were used: donkey anti-chicken IgY FITC (1:500, Merck Millipore), donkey anti-rabbit IgG647 (1:500, Life Technologies), donkey anti- rabbit IgG488 (1:500, Life Technologies) and donkey anti-mouse IgG555 (1:500, Life Technologies). Nuclei were counter stained using Hoechst (LifeTechnologies).

\section{Microfluidic chambers}

The MFCs were purchased from Xona (RD900). At first, Nunc glass bottom dishes with an inner diameter of $27 \mathrm{~mm}$ were coated with Poly-L-Ornithin over night at $37^{\circ} \mathrm{C}$. Following 3 washing steps with sterile water they were kept under the sterile hood to dry out completely. MFCs were sterilized with $70 \%$ Ethanol and also left drying. Next the MFCs were dropped on 
the glass bottom dishes and carefully pressed on the surface. Properly attached the system was then perfused with $0.01 \mathrm{mg} / \mathrm{ml}$ Laminin in PBS. After another $1 \mathrm{~h}$ at $37^{\circ}$ and one washing step with medium it could be used for cell culture. For seeding cells into this system, the entire volume was soaked out and $10 \mu \mathrm{l}$ containing a high concentration of cells $\left(15 \times 10^{\wedge} 6\right.$ cells $\left./ \mathrm{ml}\right)$ were directly injected into the main channel connecting two wells. After allowing attachment for half an hour in the incubator, the still empty wells were filled up with maturation medium. This method grants the possibility to enhance the amount of neurons growing in main channels while the wells stay free of them which reduces the medium turnover to a minimum. To avoid drying out PBS was added around the MFCs. Two days later the medium was replaced in a manner which should give the neurons a guidance cue to grow through the microchannels. Therefore a gradient style was established which was accomplished by adding $100 \mu$ N2B27 with $500 \mu \mathrm{M}$ dbcAMP to the seeding site and $200 \mu \mathrm{l}$ N2B27 with $500 \mu \mathrm{M}$ dbcAMP, $10 \mathrm{ng} / \mu \mathrm{l}$ BNDF, $10 \mathrm{ng} / \mu \mathrm{l}$ GDNF and $100 \mathrm{ng} / \mu \mathrm{l}$ NGF to the exit site. The medium composition was replaced every third day. After 7 days the first axons began spreading out at the exit site. It was possible to keep them in this system for up to 6 weeks. MFCs were analyzed by live cell imaging at 14 and 32 days post maturation induction. At day 32 they were treated with $40 \mathrm{mM}$ D-sorbitol in medium for $24 \mathrm{~h}$ following live cell imaging.

\section{Live cell imaging}

For tracking of lysosomes and mitochondria, cells were double-stained live with 50nM Lysotracker Red DND-99 (Molecular Probes Cat. No. L-7528) and 50nM Mitotracker Deep Red FM (Molecular Probes Cat. No. M22426). For measuring the mitochondrial membrane potential (and tracking as well), cells were stained with 200nM Mitotracker JC-1 (Molecular Probes Cat. No. M34152). Trackers were directly added to culture supernatants and incubated for $1 \mathrm{hr}$ at $37^{\circ} \mathrm{C}$. Imaging was then performed without further washing of cells. Live imaging of compartmentalized axons in Xona Microfluidic Chambers (MFC) was performed with a Leica HC PL APO 100x 1.46 oil immersion objective on an inversed fluorescent Leica DMI6000 microscope enclosed in an incubator chamber $\left(37^{\circ} \mathrm{C}, 5 \% \mathrm{CO} 2\right.$, humid air) and fitted with a 12 bit Andor iXON 897 EMCCD camera (512*512, 16 $\mu \mathrm{m}$ pixels, $229.55 \mathrm{~nm} /$ pixel at 100x magnification). For more details, refer to https://www.biodip.de/wiki/Bioz06__Leica_AFLX6000_TIRF. Excitation was performed with a TIRF Laser module in epifluorescence (widefield) mode with lines at $561 \mathrm{~nm}$ and $633 \mathrm{~nm}$. Fast dual color movies were recorded at 3.3 frames per second (fps) per channel over 2 min (400 frames in total per channel) with $115 \mathrm{~ms}$ exposure time as follows: Lysotracker Red (excitation: 561nm, emission filter TRITC 605/65 nm) and Mitotracker Deep Red (excitation: 633nm, emission filter Cy5 720/60 
nm). Movie acquisition was performed at strictly standardized readout positions within the micro channels of the micro groove barrier that separated the proximal seeding site from the distal axonal exit. Specifically, the readout windows were located either just adjacent to the channel exit (distal readout) or the channel entry (proximal readout).

\section{Tracking analysis}

Movies were analyzed with FIJI software using the TrackMate v2.7.4 plugin for object (lysosomes and mitochondria) recognition and tracking. Settings were as follows: Pixel width: $0.23 \mu \mathrm{m}$, Pixel height: $0.23 \mu \mathrm{m}$, Voxel Depth: $1 \mu \mathrm{m}$, Crop settings: not applied, Select a detector: DoG detector with estimated blob size: $1.6 \mu \mathrm{m}$, Threshold: 45 , median filter: no, subpixel localization: yes, Initial thresholding: none, Select view: HyperStack Displayer, Set filters on spots: quality above 45, Select a tracker: Linear Motion LAP tracker, Initial search radius. $2 \mu \mathrm{m}$, Search radius: $2 \mu \mathrm{m}$, Max. frame gap: 2 , Set filters on tracks: track duration $\geq 3 \mathrm{sec}$. Typically, 200-500 tracks per movie were obtained and analyzed with respect to track displacement (measure for processive, i.e. straight, motility as opposed to undirected random walks) and mean speed. Results were assembled and post-filtered (threshold for track displacement $\geq 1.2 \mu \mathrm{m}$ ) in KNIME and MS Excel and bulk statistics analyzed and displayed as box plots in GraphPad Prism 5 software. A minimum of 5 movies (showing 2 micro channels each) was acquired at each readout positions (distal versus proximal) per line, condition and experiment resulting in a minimum of 15 movies in total for the batch analysis.

Static analysis of cell organelles. For analysis of organelle count and morphology (mitochondria: elongation; lysosomes: diameter), object segmentation, thresholding and shape analysis was performed with a sequence of commands in FIJI software executed with Macro1 for mitochondria:

run("Clear Results"); run("Z Project...", "start=1 stop=400 projection=[Min Intensity]"); run("Enhance Contrast...", "saturated=0.1 normalize"); run("Tubeness", "sigma=0.4 use"); run("Enhance Contrast...", "saturated=0.1 normalize"); run("8-bit"); setAutoThreshold("Triangle dark"); run("Convert to Mask"); run("Skeletonize (2D/3D)"); run("Set Measurements...", "area redirect=None decimal=5"); run("Create Selection"); $\quad$ run("Measure"); $\quad$ saveAs("Measurements", $\quad$ path+name+" network.txt"); selectWindow(name); run("Slice Keeper", "first=1 last=1 increment=1"); run("Select All"); run("Set Measurements...", "kurtosis redirect=None decimal=5"); run("Clear Results"); run("Measure"); saveAs("Measurements", path+name+" Kurtosis.txt"); run("Grays"); run("Enhance Contrast...", "saturated=0.1 normalize"); run("Subtract Background...", "rolling=3"); setAutoThreshold("Moments dark"); run("Threshold..."); run("Convert to Mask"); run("Set Measurements...", "area fit shape feret's integrated area_fraction limit redirect=None decimal=5"); run("Clear Results"); run("Analyze 
Particles...", "size=4-Infinity pixel circularity=0.00-1.00 show=Ellipses clear display")

and Macro2 for lysosomes:

run("Clear Results"); run("Slice Keeper", "first=1 last=1 increment=1"); run("Grays"); run("Enhance Contrast...", "saturated=0.1 normalize"); $\quad$ run("Subtract $\quad$ Background...", $\quad$ "rolling=5"); setAutoThreshold("Moments dark"); run("Threshold..."); run("Convert to Mask"); run("Set Measurements...", "area fit shape feret's integrated area_fraction limit redirect=None decimal=5"); run("Analyze Particles...", "size=3-Infinity pixel circularity=0.00-1.00 show=Ellipses clear display")

These macros returned result tables containing the aspect ratio of fitted eclipses (long:short radius) that was taken as measure for mitochondrial elongation as well as the outer Feret's diameter that was taken as lysosomal diameter. The same set of movies as for the tracking analysis (see above) was used (first frame only). Typically, hundreds of organelles were analyzed per movie. For bulk statistics, the same batch analysis as for the tracking analysis was performed with resultant distributions displayed as box plots.

\section{Electrophysiology}

We performed patch-clamp recordings as described previously ${ }^{30}$. All electrophysiological experiments were recorded during week 7 of total differentiation. In brief, we plated 300k cells per Matrigel-coated coverslip in a 24 well plate on day 25 . To make sure we recorded from MNs we selected large (>20pF) neurons with multiple neurites only. We also filled the internal patch solution with secondary antibody Alexa 488 to allow MN identification after an additional immunostaining step which gave us $90.5 \%$ positively identified $\mathrm{MNs}^{25}$.

\section{Quantification and statistics}

Randomly assigned images of different experiments were quantified on day 14 of neuronal differentiation (=day 5 of neuron maturation) to evaluate MN differentiation capacity. Statistical evaluation was performed using 1-way ANOVA with Bonferroni post hoc test for multiple comparison. Data are depicted as mean \pm standard deviation from at least four independent experiments each.

Static and dynamic live cell imaging box plot statistics are provided in Suppl. Tables 2-9. Box plot settings: boxes from 25-75 percentile, whiskers from 1-99 percentile, outliers as dots, median as horizontal center line, mean as cross. Differences between conditions (i.e. cells lines, compound treatments, etc.) were revealed with the non-parametric Kruskal-Wallis test including Dunn's post hoc test for non-Gaussian distributions with a significance level of $\mathrm{P} \leq 0.05$ and $95 \%$ confidence interval. It was used to compute significant mean rank differences for the distribution of measured organelle property data in order to evaluate the behavior of the bulk majority of 
organelles, respectively. Box plots represent batch results merged from all mutant ALS-TDP43 lines, respectively, from at least three independent experiments. Detailed box plot statistics are presented in Supplemental Tables 2-9. 


\section{Results}

\section{Motor neuron differentiation is not affected by ALS-causing TDP43 mutations}

In order to confirm the neuronal differentiation potential and ensure that wildtype and TDP43 mutant motor neuron cultures exhibit the same differentiation capacity for further disease modeling, they were analyzed towards the proportion of neuron types, their morphology and their survival rates during motoneuronal differentiation. For this, iPSCs were derived from two different patients and three different control individuals (different families with different mutations; G294V; S393L, respectively; different controls from different families, see also Suppl. Table 1). A defined cell number of patient NPCs (neural progenitor cells) were differentiated for 9 days and subsequently seeded for the final motor neuron maturation process for 5 additional days (Figure $1 \mathrm{~A}$, schematic differentiation procedure). There was no difference in neuron morphologies between wildtype and TDP43 mutant neurons. The calculation of neuron types resulted in equal numbers of either $\beta$ III-Tubulin (pan-neuronal marker, young neurons), MAP2 (mature neurons) or SMI32 (motor neurons) positive neurons when wildtype and TDP43 mutants were compared (Figure $1 \mathrm{~B}-\mathrm{E}, P=0.107, F=2.428$ ). Nearly all neurons marked by $\beta \mathrm{III}-$ Tubulin were also positive for the mature neuron marker MAP2 (wildtypes: $96.90 \pm 1.06 \%$; TDP43 S393L: 98.5 $\pm 3.0 \%$; TDP43 G294V: 100.5 \pm 1.0$)$. The majority of mature MAP2 ${ }^{+}$ neurons were also identified as $\mathrm{SMI}_{3}{ }^{+}$spinal motor neurons in wildtypes and TDP43 mutants

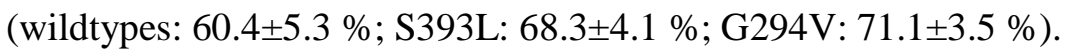

Total cell death and the number of dying neurons (marked by pyknotic cell nuclei, Figure $1 \mathrm{G}, \mathrm{H}$ ) revealed a significantly increased total cell death in TDP43 G294V mutant motor neuron cultures $(46.8 \pm 6.1 \%)$ when compared to wildtype $(17.9 \pm 2.4 \%, P<0.0001)$ and TDP43 S393L mutant $(24.8 \pm 3.1 \%, P=0.0007)$. The death of young- ( $\beta$ III-Tubulin), mature- (MAP2) or spinal motor neurons (SMI32) was not differing when wildtype and TDP43 mutants were compared.

Next we determined growth behavior and metabolism: Counting of Hoechst ${ }^{+}$nuclei as well as metabolic activity and LDH release measurement showed no difference in growth of wildtype and TDP43 mutant motor neuron cultures (Figure 1 H-J, Hoechst: $P=0.959, F=0.041$; metabolism: $P=0.126, F=2.513$; LDH: $P=0.130, F=2.341$ ).

Together the early motor neuron maturation analysis showed an efficient homogeneous neuron differentiation with a high spinal motor neuron differentiation potential $\left(\mathrm{SMI}_{3}{ }^{+} / \mathrm{CHAT}^{+}\right.$, Figure $1 \mathrm{C})$ in all analyzed motor neuron cultures.

Furthermore, both, wildtype and TDP43 mutant iPSC-derived motor neurons acquired normal neuronal function such as sodium and potassium currents (Figure 2 A-D), single and repetitive 
action potentials (Figure $2 \mathrm{E}, \mathrm{F}$ ) as well as spontaneous postsynaptic currents (G-I) with no significant differences between healthy and TDP43 mutant motor neurons.

\section{Mutant TDP43 motor neurons show age-related disturbed distal axonal organelle morphology}

During aging mitochondria and lysosomes were visualized by Mito- and Lysotracker labelling of motor neuron cultures in directed microfluidic chambers (MFCs) at 2 and 4 weeks of maturation/aging (Figure 1 A \& 3 A, B). Comparing wildtype and TDP43 mutants, measurement of neurite length variations from young to aged stages (i.e. fold-change over corresponding younger time point) revealed a constant proximal neurite mitochondrial network size for all lines whereas at the distal site wildtype cells exhibited a clear 1,179 -fold increase $( \pm 0,194)$ as opposed to a moderate 0.721 -fold decrease $( \pm 0,070)$ in the mutants $(P=0.004)$ (Figure $3 \mathrm{D})$. Considering the number of measured organelle objects it could be excluded that the differences in neurite network size arose from different lysosome or mitochondria numbers (Figure 3 D; lysosomes: $P=0.928$; mitochondria: $P=0.545)$. Moreover, there was no difference in the mitochondrial membrane potential between wildtypes and TDP43 mutant neurons (Figure $3 \mathrm{E}$ ).

Mitochondria and lysosomes were both analyzed with respect to their individual organelle size and shape properties (Figure $3 \mathrm{~F}-\mathrm{I})$. The study of the mitochondrial area (=size) revealed a distal phenotype of aged TDP43 mutant MFC motor neurons whereas mitochondria at the proximal axon areas showed no differences. The analysis of lysosomal area resulted solely in a moderate distal phenotype of aged TDP43 mutant cultures when compared to young ones (Figure 3 G; detailed statistics are provided in Suppl. Tables 2 and 3). Together, the size of the majority of mitochondria and lysosomes were exclusively affected at distal axon sites of aged TDP43 mutant motor neuron cultures.

Next, we investigated mitochondrial and lysosomal shape (Figure $3 \mathrm{H}, \mathrm{I}$ ). Our analysis of the mitochondrial aspect ratio (AR; indicates the shape ranging from round $(\mathrm{AR}=1)$ to elongated $(\mathrm{AR}>1)$ morphologies; long:short radius) revealed diverse phenotypes differing from proximal to distal axon sites (Figure $3 \mathrm{H}$ ). At proximal axons, we observed a mitochondrial shortening from young to aged cultures in both wildtype and TDP43 mutants that was also present in distal axons of wildtype neurons (proximal: 2.34 $\pm 0.03, P<0.0001$; distal: $2.54 \pm 0.03, P=0.003$; Detailed statistics are presented in Suppl. Table 4). Taken together, the majority of mitochondria of TDP43 mutant MFC cultures revealed a global phenotype of imbalanced shape properties when proximal and distal axon sites were compared and in contrast to wildtype they got elongated during aging. 
Furthermore, analyzing the Feret diameter of lysosomes (i.e. the diameter of the outer organelle's perimeter) revealed a significant proximal decrease in TDP43 mutant motor neurons from young to aged stages as opposed to the increase in the wildtype during the same time. (Figure 3 I; detailed statistics are provided in Suppl. Table 5). Conversely, at the distal end we revealed a more vague trend towards smaller lysosome diameters in all lines.

\section{TDP43 mutant motor neurons show a dramatic loss of organelle motility during aging}

The motility of mitochondria and lysosomes is a critical property especially in neurons since it mainly determines the energy supply and cellular waste removal of cells. We analyzed the organelle mean speed and the extent of organelle displacement in MFC motor neuron cultures and identified a drastic global (i.e. at either proximal and distal site) axonal transport deficiency in TDP43 mutants in aging but not young motor neurons (Figure 4 A; Suppl. Videos 1 \& 2).

In detail, mitochondria of TDP43 mutant neurons exhibited a drastic slow-down of mitochondrial mean speed at proximal and distal axons during aging (Figure 4 A, B; Suppl. Videos 1, 2; Suppl. Table 6). Consistently, we revealed a decrease of mitochondrial displacement (=extent of movement radius, measure for processivity in motility) (Figure $4 \mathrm{D}$, for detailed statistics see Suppl. Table 6; 8).

The analysis of lysosomal mean speed uncovered similar trafficking defects (Suppl. Videos 1; 2): lysosomes of TDP43 mutant neurons significantly decreased their mean speed (Figure 4 A, C) and displacement (Figure 4 E, for detailed statistics see Suppl. Table 7, 9) at both axon sites during aging. Overall, our data indicate a gross decrease of mitochondrial and lysosomal organelle motility during aging at both distal and proximal axons in TDP43 mutant motor neurons.

\section{Aged TDP43 mutant motor neuron cultures show increased neuron death without signs of TDP43 protein aggregation formation}

The neuronal homogeneity during the early neuron maturation stage (Figure 1) provided an ideal basis to study the cellular development of aging wildtype and TDP43 mutant motor neurons at later time points as analyzed by live cell imaging. In order to investigate if the used human model systems in this study recapitulate ALS hallmarks of neuron death and degeneration the survival of neurons including spinal motor neurons was investigated at different time points during cellular aging. The analysis from young (day 5 of maturation) to aged (day 32 of maturation) mutant cultures showed a significant loss in the total amount of $\beta$ III-Tubulin (Figure 5 A; day 5: $53.69 \pm 4.83 \%$, day 32: $19.53 \pm 3.14 \%$; $P<0.0001$ ), MAP2 (Figure 5 B; day 5: $53.49 \pm 5.95 \%$, day 32: $33.40 \pm 5.93 \% ; P=0.022$ ) and SMI32 positive neurons (Figure $5 \mathrm{C}$; day 
5: $34.67 \pm 4.10 \%$, day $32: 5.0 \pm 1.05 \% ; P<0.0001)$ whereas in the wildtype this loss was only moderate (Figure 5 A-C; day 32: $\beta$ III-Tubulin: $52.51 \pm 6.94 \%, P=0.0002$; MAP2: $56.03 \pm 3.62$ $\%, P=0.013$; SMI32: $15.93 \pm 2.50 \%, P=0.004)$. Together, our results document a clear vulnerability and compromised survival in TDP43 mutant spinal motor neurons during cellular aging whereas wildtype cells could maintain a constant neuron pool. Interestingly, the agedependent increase of neuronal death was not accompanied by TDP43 protein aggregation (Figure 1 D). Wildtype as well as TDP43 mutant cells showed a dominant nuclear localization of TDP43 protein and no detectable phosphorylated TDP43(Supplemental Figure 1).

\section{TDP43 mutant neurons show dramatic axo-skeletal degeneration during aging}

The analysis of cytoskeletal alterations during neuronal aging revealed dramatic differences between wildtype and TDP43 mutant motor neuron cultures (Figure 5 D-I). We analyzed tubulin ( $\beta$ III-Tubulin), microtubule-associated protein Tau (MAP) as well as neurofilament-H (NF-H, SMI32). Cytoskeletal proteins of wildtype neurons appeared as firm, smooth and thick (indicated as "smooth"). In contrast, the cytoskeleton of TDP43 mutant neurons exhibited disrupted morphologies of shortened and fragile dendrites (MAP2) and axons (SMI32) combined with striking loss of neurites and branching (indicated as "disrupted"). A third structural morphology change identified as smooth filament with intermittently occurring filament beads (indicated as "beady") could be detected in wildtype cells and TDP43 mutants. A schematic demonstration is given in Figure $5 \mathrm{E}$ and representative images are shown in Figure 5 I. Morphologically aberrant neurons were quantified at day 32 of maturation (Figure $5 \mathrm{E}-\mathrm{H}$ ) and the cytoskeleton structures were classified by their appearance (smooth, beady and disrupted; Figure 5 E). Collectively, our findings document an age- and TDP43-mutation-related abnormal cytoskeletal morphology in mature spinal motor neurons.

\section{D-Sorbitol rescues mutant TDP43 motor neuron organelle morphology and motility without inducing TDP43 aggregation}

Osmolytes are small organic compounds that affect protein stability and are ubiquitous in living systems. D-sorbitol is known as protein protecting and stabilizing osmolyte ${ }^{31}$. Furthermore, Dsorbitol was reported to recruit TDP43 to stress granules ${ }^{18}$, to induce cytoplasmic mislocalization and to interfere with the quality control machinery in TDP43 mutants ${ }^{19}$. To further address these findings, we treated aged MFC motor neuron cultures with $40 \mathrm{mM}$ up to $400 \mathrm{mM}$ of D-sorbitol for $24 \mathrm{~h}$. Interestingly, D-sorbitol did not induce any cytoplasmic TDP43 protein localization or aggregation (Figure 6 K, L; Supplement Figure 2). 
Very surprisingly, however, we found a profound rescue of organelle morphology and deficient motility in mutant TDP43 motor neurons by D-sorbitol treatment (40 mM). Specifically, Dsorbitol caused a significant increase of the distal axon TDP43 mutant network size (Figure 6 A, B; untreated: 117.2 \pm 2.93 pixel $^{2}$, D-sorbitol: 168.0 \pm 5.62 pixel2 $\left.^{2} P<0.0001\right)$. Considering the single organelle size the treatment with D-sorbitol eliminated the soft differences in distal mitochondria size between aged wildtype and TDP43 mutant MFC cultures (Figure $6 \mathrm{C}$; treated wildtype: $55.3 \pm 2.89$ pixel $^{2}$, treated TDP43 mutants: $65.0 \pm 3.35$ pixel $^{2} ; P>0.999$ ). For lysosomes it led to a restoration of the young distal TDP43 mutant morphology (see Figure $3 \mathrm{G}$ ) by highly increased lysosomal organelle area mean to $79.24 \pm 4.08$ pixel$^{2}$ (Figure $6 \mathrm{G}$; untreated: $54.57 \pm 1.83$ pixel $\left.^{2}, \mathrm{P}<0.0001\right)$ whereas the wildtype stayed unaffected. Together D-sorbitol affected organelle sizes only at distal axon sites whereas the organelle shape and motility were influenced at both axon sites.

Additionally, D-sorbitol showed a clear rescue of organelle motility (Figure 6 E, F, I and J and Suppl. Videos $3 \& 4$ ), i.e. mitochondrial speed (Figure 6 E) and displacement (Figure 6 F), as well as lysosomal speed (Figure $6 \mathrm{I}$ ) and displacement (Figure $6 \mathrm{~J}$; for detailed statistics see Suppl. Tables 2 - 9). 


\section{Discussion}

Here we show age-dependent neurodegeneration of patient-derived TDP43 mutant motor neurons similar to Bilican and colleagues ${ }^{12}$. Furthermore, we present substantial data arguing against TDP43 aggregation as upstream event in the pathophysiology of TDP43-ALS. Initially, morphologically healthy appearing motor neurons (Figure 1, similarly reported in ${ }^{11}$ ) revealed robust TDP43-mutation-dependent abnormal phenotypes of mitochondria and lysosomes (Figures 3, 4, 6) and the cytoskeleton (Figure 5) during aging without signs of cytoplasmic TDP43 aggregation (Figures 1, 5).

Since the first description of a motor neuron disease a well-known hallmark in the pathology is progressive motor neuron degeneration in the spinal cord ${ }^{32}$ and, by modern neuropathology techniques, pathogenic TDP43 aggregation in motor neurons of ALS and FTLD ${ }^{5,33}$ and other neurodegenerative disorders ${ }^{34}$. In this study, we document pathological degeneration of in vitro cultivated patient-derived (iPSCs) spinal motor neurons of TDP43 mutation carriers characterized by dramatic neuron and spinal motor neuron loss during aging and the formation of severely abnormal cytoskeletal structures in mutant cultures (Figure 5). In contrast, we could not observe cytoplasmic TDP43 localization or aggregation in aged neurons and spinal motor neurons (Figure 1,5) assuming a TDP43 protein mislocalization-independent mechanism of neurodegeneration. We cannot rule out that the mutations used in the current study behave slightly different to other TDP43 mutations ${ }^{35}$. However, our data is not in contrast to recent studies reporting cytosolic mislocalization of TDP43 since, on a closer look, also in these models suing different TDP43 mutations only up to one third of cells showed cytosolic mislocalization while the remaining cells still showed physiological nuclear TDP43 ${ }^{10,11,14}$.

Surprisingly, we could not detect relevant cytoplasmic TDP43 mislocalization or aggregation even after treatment with the osmolyte D-sorbitol being reported to induce stress granules formation and cytoplasmic mislocalization of TDP43 ${ }^{18}$. Furthermore, D-sorbitol rescued organelle morphology and motility in TDP43 mutant patient-derived neurons (Figure 5). Neurite outgrowth was reported being reduced in murine models of TDP43 ${ }^{9,20}$ which could be restored by non-toxic proteasome inhibition ${ }^{20}$. D-sorbitol is known as protein protecting and stabilizing osmolyte ${ }^{31}$. This could explain our rather unexpected results showing that the osmolyte sorbitol leads to restoration of the axon trafficking phenotype. Together, both results argue against a TDP43 aggregation-dependent mechanism as early event in neurodegeneration of TDP43 motor neuron degeneration.

The size of the majority of mitochondria and lysosomes was mainly affected at distal axon parts (directed to axon endings) indicated by elongated mitochondria and shrunken lysosomes in comparison to wildtype cases (Figure $3 \& 6$ ). Furthermore, we detected in aged but 
not young TDP43 mutant motor neurons an overall organelle trafficking deficit (Figure 4 \& 6). This is in accordance with TDP43 mice model data showing lack of mitochondria in motor axon terminals ${ }^{10}$. Furthermore, TDP43 was reported to impair mitochondria and lysosome morphology and function in cell models of ALS. Interestingly, both overexpression and knockdown caused a phenotype in primary murine neurons suggesting a tight regulation of TDP43 ${ }^{22,23}$.

TDP43 was shown to be actively transported in the axon thereby forming cytoplasmic mRNP granules that undergo bidirectional, microtubule-dependent transport in neurons and facilitate delivery of target mRNA to distal neuronal compartments ${ }^{14,15}$. TDP43 mutations impair this mRNA transport function ${ }^{14,15}$. Several lines of evidence suggest that mRNA encoding cytoskeletal proteins is cargo of such mRNA-transporting proteins being locally translated in the distal axon ${ }^{36,37}$. For instance, neurofilament light chain (NEFL) mRNA was shown to be transported in such TDP43 granules and was reduced in TDP43 mutant motor neurons ${ }^{10,15}$. These data fit to our observations of severely disturbed cytoskeletal proteins such as tubulin, microtubules and neurofilaments. Furthermore, this could induce a vicious cycle by additionally impairing microtubule-dependent mRNP granule transport ${ }^{14,15}$.

Together, we here report typical age-related motor neurodegeneration in TDP43 mutant ALS patient-derived motor neurons leading to a severe organelle transport deficiency, cytoskeletal disturbance and neurodegeneration without TDP43 protein mislocalization or aggregation formation. This challenges the current concept of protein aggregation as the main cause of motor neuron disease pathology and calls for a search for therapeutic targets independent of TDP43 aggregation.

\section{Conclusions}

We here show aging-related neurodegeneration in patient specific TDP43 mutant iPSC-derived motor neuron cultures. Cell organelles as mitochondria and lysosomes showed TDP43 mutation dependent abnormalities in size, shape, speed and motility which all appeared without TDP43 mislocalization or aggregation formation. Finally, D-sorbitol - known to induce stress granules and cytoplasmic mislocalization of TDP43 - rescued axonal trafficking phenotypes without any signs of TDP43 mislocalization or aggregation formation. Our data suggest TDP43 mutation dependent but cytosolic aggregation -independent mechanisms of motor neuron degeneration in TDP43 ALS. 


\section{Additional information}

\section{Authors' contributions}

Nicole Kreiter, geb. Wächter: Conception and design, collection and assembly of data, data analysis and interpretation, manuscript drafting.

Arun Pal: Data collection and assembly, partial analysis and interpretation, manuscript drafting.

Xenia Lojewski: Generation of iPS and derivation of NPCs from patient fibroblasts, manuscript drafting.

Philippe Corcia: TDP43 mutant patient fibroblast delivery, manuscript drafting.

Maximilian Naujock: Data collection and assembly, partial analysis and interpretation, manuscript drafting.

Peter Reinhardt: Generation of iPS and derivation of NPCs from patient fibroblasts, manuscript drafting.

Jared Sterneckert: Generation of iPS and derivation of NPCs from patient fibroblasts, manuscript drafting.

Susanne Petri: Data collection and assembly, partial analysis and interpretation, manuscript drafting.

Florian Wegner: Data collection and assembly, partial analysis and interpretation, manuscript drafting.

Alexander Storch: Conception and design, data interpretation, manuscript drafting.

Andreas Hermann: Conception and design, principal investigator, collection and assembly of data, data interpretation, manuscript drafting and final approval.

\section{Competing financial interests}

The authors declare that they have no conflict of interest.

\section{Acknowledgements}

We thank the patients and controls for skin biopsy donation. We acknowledge the help in cell culture by Sylvia Kanzler and Katja Zoschke. The work was supported in part by the Helmholtz Virtual Institute (VH-VI-510) "RNA dysmetabolism in ALS and FTD" for the funding of the project to A.H. and A.S., the "Deutsche Gesellschaft für Muskelerkrankungen (He 2/2)", the MeDDrive program of the Medical Faculty at the Technische Universität Dresden to A.H., the Center for Regenerative Therapies Dresden, the Roland Ernst Stiftung Saxony to A.H., BIOCREA GMBH to A.H. and the NOMIS foundation to A.H.. 


\section{References}

1 Ludolph, A. C. \& Sperfeld, A. D. Preclinical trials--an update on translational research in ALS. Neuro-degenerative diseases 2, 215-219, doi:10.1159/000089628 (2005).

2 Johnston, C. A. et al. Amyotrophic lateral sclerosis in an urban setting: a population based study of inner city London. Journal of neurology 253, 1642-1643, doi:10.1007/s00415-006-0195-y (2006).

3 Rosen, D. R. et al. Mutations in $\mathrm{Cu} / \mathrm{Zn}$ superoxide dismutase gene are associated with familial amyotrophic lateral sclerosis. Nature 362, 59-62, doi:10.1038/362059a0 (1993).

4 Robberecht, W. \& Philips, T. The changing scene of amyotrophic lateral sclerosis. Nat Rev Neurosci 14, 248-264, doi:10.1038/nrn3430 (2013).

5 Neumann, M. et al. Ubiquitinated TDP-43 in frontotemporal lobar degeneration and amyotrophic lateral sclerosis. Science 314, 130-133, doi:10.1126/science.1134108 (2006).

6 Scotter, E. L., Chen, H. J. \& Shaw, C. E. TDP-43 Proteinopathy and ALS: Insights into Disease Mechanisms and Therapeutic Targets. Neurotherapeutics : the journal of the American Society for Experimental NeuroTherapeutics 12, 352-363, doi:10.1007/s13311015-0338-x (2015).

7 Mackenzie, I. R. et al. Pathological TDP-43 distinguishes sporadic amyotrophic lateral sclerosis from amyotrophic lateral sclerosis with SOD1 mutations. Annals of neurology 61, 427-434 (2007).

8 Jovicic, A. \& Gitler, A. D. TDP-43 in ALS: stay on target...almost there. Neuron 81, 463465, doi:10.1016/j.neuron.2014.01.034 (2014).

9 Han, J. H. et al. ALS/FTLD-linked TDP-43 regulates neurite morphology and cell survival in differentiated neurons. Exp Cell Res 319, 1998-2005, doi:10.1016/j.yexcr.2013.05.025 (2013).

10 Shan, X., Chiang, P. M., Price, D. L. \& Wong, P. C. Altered distributions of Gemini of coiled bodies and mitochondria in motor neurons of TDP-43 transgenic mice. Proc Natl Acad Sci U S A 107, 16325-16330, doi:10.1073/pnas.1003459107 (2010).

11 Zhang, Z. et al. Downregulation of microRNA-9 in iPSC-derived neurons of FTD/ALS patients with TDP-43 mutations. PLoS One 8, e76055, doi:10.1371/journal.pone.0076055 (2013).

12 Bilican, B. et al. Mutant induced pluripotent stem cell lines recapitulate aspects of TDP43 proteinopathies and reveal cell-specific vulnerability. Proc Natl Acad Sci U S A 109, 5803-5808, doi:10.1073/pnas.1202922109 (2012).

13 Nishimura, A. L. et al. Allele-specific knockdown of ALS-associated mutant TDP-43 in neural stem cells derived from induced pluripotent stem cells. PLoS One 9, e91269, doi:10.1371/journal.pone.0091269 (2014).

14 Fallini, C., Bassell, G. J. \& Rossoll, W. The ALS disease protein TDP-43 is actively transported in motor neuron axons and regulates axon outgrowth. Hum Mol Genet 21, 3703-3718, doi:10.1093/hmg/dds205 (2012).

15 Alami, N. H. et al. Axonal transport of TDP-43 mRNA granules is impaired by ALScausing mutations. Neuron 81, 536-543, doi:10.1016/j.neuron.2013.12.018 (2014).

16 Liu-Yesucevitz, L. et al. Tar DNA binding protein-43 (TDP-43) associates with stress granules: analysis of cultured cells and pathological brain tissue. PLoS One 5, e13250, doi:10.1371/journal.pone.0013250 (2010).

17 Freibaum, B. D., Chitta, R. K., High, A. A. \& Taylor, J. P. Global analysis of TDP-43 interacting proteins reveals strong association with RNA splicing and translation machinery. J Proteome Res 9, 1104-1120, doi:10.1021/pr901076y (2010).

18 Dewey, C. M. et al. TDP-43 is directed to stress granules by sorbitol, a novel physiological osmotic and oxidative stressor. Mol Cell Biol 31, 1098-1108, doi:10.1128/MCB.01279-10 (2011). 
19 Zhang, T., Baldie, G., Periz, G. \& Wang, J. RNA-processing protein TDP-43 regulates FOXO-dependent protein quality control in stress response. PLoS Genet 10, e1004693, doi:10.1371/journal.pgen.1004693 (2014).

20 Duan, W., Guo, Y., Jiang, H., Yu, X. \& Li, C. MG132 enhances neurite outgrowth in neurons overexpressing mutant TAR DNA-binding protein-43 via increase of HO-1. Brain Res 1397, 1-9, doi:10.1016/j.brainres.2011.05.006 (2011).

21 Wächter, N., Storch, A. \& Hermann, A. Human TDP-43 and FUS selectively affect motor neuron maturation and survival in a murine cell model of ALS by non-cellautonomous mechanisms. Amyotrophic lateral sclerosis \& frontotemporal degeneration 16, 431-441, doi:10.3109/21678421.2015.1055275 (2015).

22 Wang, W. et al. The ALS disease-associated mutant TDP-43 impairs mitochondrial dynamics and function in motor neurons. Hum Mol Genet 22, 4706-4719, doi:10.1093/hmg/ddt319 (2013).

23 Xia, Q. et al. TDP-43 loss of function increases TFEB activity and blocks autophagosome-lysosome fusion. EMBO J 35, 121-142, doi:10.15252/embj.201591998 (2016).

24 Japtok, J. et al. Stepwise acquirement of hallmark neuropathology in FUS-ALS iPSC models depends on mutation type and neuronal aging. Neurobiology of disease 82, 420429, doi:10.1016/j.nbd.2015.07.017 (2015).

25 Naujock, M. et al. 4-Aminopyridine Induced Activity Rescues Hypoexcitable Motor Neurons from Amyotrophic Lateral Sclerosis Patient-Derived Induced Pluripotent Stem Cells. Stem Cells 34, 1563-1575, doi:10.1002/stem.2354 (2016).

26 Reinhardt, P. et al. Derivation and expansion using only small molecules of human neural progenitors for neurodegenerative disease modeling. PLoS One 8, e59252, doi:10.1371/journal.pone.0059252 (2013).

27 Lojewski, X. et al. Human iPSC models of neuronal ceroid lipofuscinosis capture distinct effects of TPP1 and CLN3 mutations on the endocytic pathway. Hum Mol Genet 23, 2005-2022, doi:10.1093/hmg/ddt596 (2014).

28 Stanslowsky, N. et al. Neuronal Dysfunction in iPSC-Derived Medium Spiny Neurons from Chorea-Acanthocytosis Patients Is Reversed by Src Kinase Inhibition and F-Actin Stabilization. J Neurosci 36, 12027-12043, doi:10.1523/JNEUROSCI.0456-16.2016 (2016).

29 DeJesus-Hernandez, M. et al. Expanded GGGGCC hexanucleotide repeat in noncoding region of C9ORF72 causes chromosome 9p-linked FTD and ALS. Neuron 72, 245-256, doi:10.1016/j.neuron.2011.09.011 (2011).

30 Naujock, M. et al. Molecular and functional analyses of motor neurons generated from human cord-blood-derived induced pluripotent stem cells. Stem Cells Dev 23, 3011-3020, doi:10.1089/scd.2014.0180 (2014).

31 Street, T. O., Bolen, D. W. \& Rose, G. D. A molecular mechanism for osmolyte-induced protein stability. Proc Natl Acad Sci $U$ S A 103, 13997-14002, doi:10.1073/pnas.0606236103 (2006).

32 Charcot, J. M. \& Joffory, A. Deux cas d'atrophie musculaire progressive avec lesions de la substance grise et des faisceaux antero-lateraux de la moelle epiniere. Arch. Physiol. Neurol. Pathol. 2, 744-754 (1869).

33 Arai, T. et al. TDP-43 is a component of ubiquitin-positive tau-negative inclusions in frontotemporal lobar degeneration and amyotrophic lateral sclerosis. Biochem Biophys Res Commun 351, 602-611, doi:10.1016/j.bbrc.2006.10.093 (2006).

34 Lee, E. B., Lee, V. M. \& Trojanowski, J. Q. Gains or losses: molecular mechanisms of TDP43-mediated neurodegeneration. Nature reviews. Neuroscience 13, 38-50, doi:10.1038/nrn3121 (2012).

35 Origone, P. et al. Enlarging clinical spectrum of FALS with TARDBP gene mutations: S393L variant in an Italian family showing phenotypic variability and relevance for 
genetic counselling. Amyotroph Lateral Scler 11, 223-227, doi:10.3109/17482960903165039 (2010).

36 Rossoll, W. et al. Smn, the spinal muscular atrophy-determining gene product, modulates axon growth and localization of beta-actin mRNA in growth cones of motoneurons. $J$ Cell Biol 163, 801-812, doi:10.1083/jcb.200304128 (2003).

37 Glinka, M. et al. The heterogeneous nuclear ribonucleoprotein-R is necessary for axonal beta-actin mRNA translocation in spinal motor neurons. Hum Mol Genet 19, 1951-1966, doi:10.1093/hmg/ddq073 (2010).

38 Vance, C. et al. Mutations in FUS, an RNA processing protein, cause familial amyotrophic lateral sclerosis type 6. Science 323, 1208-1211, doi:10.1126/science.1165942 (2009). 


\section{Figure legends}

Figure 1: Characterization of spinal motor neuron differentiation potential. (A) Human spinal motor neuron differentiation steps. Black arrowheads indicate splits. Analysis (*) was performed at NPC stage and at several stages after re-seeding of the motor neuron cultures for maturation. (B) Representative immunofluorescence stainings of neurons generated from wildtype and TDP43 mutant patient NPCs 5 days post maturation induction. Shown are the panneuronal marker $\beta$ III-Tubulin, the mature neuron marker MAP2 and the spinal motor neuron marker SMI32. There was no difference in neuron morphology between wildtype and TDP43 mutant neuron types. Cell nuclei were stained with Hoechst. Scale bars: $75 \mu \mathrm{m}$. (C) Representative immunofluorescence stainings of mature motor neurons (SMI32) positive for the cholinergic spinal motor neuron marker choline acetyltransferase (CHAT). Cell nuclei were stained with Hoechst. Scale bars: $25 \mu \mathrm{m}$. (D) SMI32+ motor neurons showed no cytoplasmic accumulation of TDP43 protein. Scale bars: $25 \mu \mathrm{m}$. (E) Quantitative analysis of total $\beta$ IIITubulin, MAP2/BIII-Tubulin and SMI32/MAP2 positive neuron soma as percentage of total living cell number (intact Hoechst $(n=4)$. (F) Quantitative analysis of total cell death, $\beta$ IIITubulin $^{+}$neuron death, MAP2 ${ }^{+}$neuron death and SMI32 ${ }^{+}$motor neuron death as percentage of total dying cell number $(n=4)$; pyk. - pyknotic. (G) Quantitative analysis of the survival- and death rates of cells in wildtype and TDP43 mutant motor neuron cultures revealed increased death rates in TDP43 G294V mutant cultures when normalized to the initially seeded cell number $(n=4)$. (H) Quantitative data of Hoechst ${ }^{+}$nuclei count per image showed no differences in cell numbers between wildtypes and TDP43 mutant cultures $(n=4-7)$. (I) Metabolic activity measurement by PrestoBlue ${ }^{\circledR}$ assay resulted in similar behavior comparing wildtype and TDP43 mutant neuron culture metabolism $(n=4-6)$. (J) Determination of cell damage by LDH activity measurement revealed no discrepancies between the wildtype and TDP43 motor neuron cultures $(\mathrm{n}=5-8)$. (E-J) Bar graphs show mean with SEM (1-way ANOVA (G-J) and 2-way ANOVA (E, F) with Bonferroni post hoc test, $* P \leq 0.05, * * P \leq 0.01, * * * P \leq 0.001$, **** $P \leq 0.0001)$.

\section{Figure 2: Functional maturation of healthy control and TDP43 mutant iPSC-derived motor}

neurons. (A) Stepwise depolarization in $10 \mathrm{mV}$ increments from a holding potential of $-70 \mathrm{mV}$ to $40 \mathrm{mV}$ revealed voltage-dependent potassium and sodium currents (B) which were normalized for each respective cell capacitance. (C) No significant differences were observed in either potassium or sodium current peaks (D) nor sodium to potassium current ratios $\left(\mathrm{Na}^{+} / \mathrm{K}^{+}\right)$when comparing healthy control $(n=127)$ to TDP43 mutant $(n=34)$ iPSC derived neurons. (E) At least single (sAP) as well as repetitive trains of action potentials (rAP) were observed in the majority 
of the recorded neurons without a significant difference between groups (F). (G) Spontaneous activity was observed as post-synaptic currents (PSCs) and action potentials (APs). No differences between control and TDP43 mutant neurons were observed in the (H) percentage or (I) frequency of PSCs and APs.

\section{Figure 3: Cell organelle morphology and neurite network size during cellular aging. (A, B)}

Schematic illustration of analyzed proximal and distal sites of a motor neuron axon and representative images taken from the live cell movies of Mitotracker (upper panel, yellow), Lysotracker (middle panel, turquoise) and Mitotracker JC-1 (lower panel, red) in wildtype (WT), TDP43 S393L and G294V mutant microfluidic chamber (MFC) motor neuron cultures . Images show one passed MFC channel and display sections from 100x magnifications. Scale bars: 10 $\mu \mathrm{m}$. (C) The proximal neurite network size (fold-change of aged cultures over corresponding young time point) did not differ between wildtype or TDP43 mutants. Conversely, the distal neurite network size decreased in the mutants over time as opposed to the increase in the wildtype. (D) The number of tracked organelles showed no difference between wildtype and TDP43 mutants. (E) No difference was seen in mitochondrial membrane potential. (D-E) Data of TDP43 mutants were pooled. Bar graphs show mean with SEM (n=3, 1-way ANOVA with

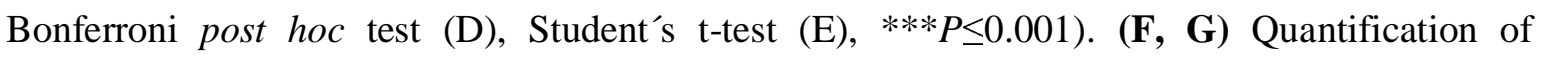
organelle size of young and aged mitochondria $(F)$ and lysosomes $(G)$ revealed a severe distal mitochondrial phenotype in TDP43 mutant cultures characterized by increased size. (H, I) Quantification of organelle shape of young and aged mitochondria ( $\mathrm{H}$, aspect ratio) and lysosomes (I, Feret's diameter) revealed significantly elongated mitochondria (increased aspect ratio) at both axon sites of aged TDP43 mutant neurons. (F-I) Data of TDP43 mutants were pooled. Box plots show mean (+) and median (line) and whiskers from 1-99 percentile ( $\mathrm{n}=3-5$, Kruskal-Wallis test with Dunn's post hoc test; asterisks compare cell lines (e.g. wt/TDP43 mutants) within a condition (e.g. distal/proximal), rhombs compare between conditions within a cell line; $\left.{ }^{* / \#} P \leq 0.05, * * / \# \# P \leq 0.01, * * * P \leq 0.001, * * * * \# \# \#+1 \leq 0.0001\right)$. For detailed statistics see Suppl. Tables 2 - 5.

Figure 4: Cell organelle motility during cellular aging. (A) Shown are maximum intensity projections of entire movie stacks. Processively moving organelles (Mito-/Lysotracker) appear as continuous trajectories (bright lines) whereas less motile organelles as shorter lines and dots. (B, C) Organelle speed analysis of young and aged mitochondria (B) and lysosomes (C) compared in proximal and distal axon parts revealed a dramatic speed loss of either organelle type and 
axon sites in TDP43 mutant neurons. (D, E) Displacement analysis of young and aged mitochondria (D) and lysosomes (E) at proximal and distal axon sites revealed a dramatic decrease in processive motility of either organelle types and axon sites in aging TDP43 mutant motor neurons. (B-E) Data of TDP43 mutants were pooled. Box plots show mean (+) and median (line) and whiskers from 1-99 percentile (n=3-5, Kruskal-Wallis test with Dunn's post hoc test; asterisks compare cell lines (e.g. wt/TDP43 mutants) within a condition (e.g. distal/proximal), rhombs compare between conditions within a cell line; $* P \leq 0.05, * * P \leq 0.01$, $\left.* * * P \leq 0.01, * * * *{ }^{\prime \# \# \#} P \leq 0.0001\right)$. For detailed statistics see Suppl. Tables 6-9.

Figure 5: The TDP43 mutation related loss of neurons during aging is accompanied by abnormal neurofilament morphologies. (A-C) The total number of $\beta$ III-Tubulin (A), MAP2 (B) and SMI32 (C) positive neurons was counted in young (day 5) and aged (day 32) maturation cultures and revealed a severe loss of every type of neuron in aged TDP43 mutant cultures, ( $n=6$ 8). (D) The number of abnormal neurons was evaluated in aged cultures and revealed a highly increased number of misshaped $\beta$ III-Tubulin, MAP2 and SMI32 positive neurons indicated by beady (b) and disrupted (d) cytoskeleton morphologies. (E) Schematic demonstration of a neuron forming smooth (s), beady (b) and disrupted (d) cytoskeleton morphologies, (n=3-8). (F-H) The number of neurons showing smooth, beady or disrupted cytoskeleton per neuron type was calculated and revealed a dramatic loss of normal smooth cytoskeleton morphology for MAP2 (G) and SMI32 (H) positive mature neurons in TDP43 mutants, consistent with a drastic increase of disrupted cytoskeleton morphology in all neuron types in TDP43 mutants (n=6-8). (I) Representative immunofluorescence images of cytoskeletal morphologies (smooth: hollow white arrow heads, beady: filled white arrow heads, disrupted: filled yellow arrow heads) of 32 days maturated wildtype and TDP43 mutant motor neurons. Cell nuclei were stained with Hoechst. Images display sections of 20x magnifications. Scale bars: $25 \mu \mathrm{m}$. (A-D and F-H) Data of wildtype 1-3 as well as TDP43 mutant lines were pooled. Bar graphs show mean with SEM (2way ANOVA with Bonferroni post hoc test, $* \mathrm{P} \leq 0.05$, **P $\leq 0.01, * * * \mathrm{P} \leq 0.001$, **** $\mathrm{P} \leq 0.0001)$.

\section{Figure 6: Analysis of cell organelles in aged spinal motor neuron cultures after 24 h D-}

sorbitol treatment. (A) Representative images taken from live cell movies of Mitotracker (left) and Lysotracker (right) ${ }^{38}$ in D-sorbitol-treated wildtype (WT), TDP43 S393L and G294V mutant MFC motor neuron cultures. Images show one passed MFC channel at proximal and distal sites and display sections from 100x magnifications. Scale bars: $10 \mu \mathrm{m}$. (B) Reduced neurite network size in aged TDP43 mutants at the distal axon site was restored by the treatment with 40mM Dsorbitol. (C, G) Proximal and distal organelle sizes of mitochondria (C) and lysosomes (G) from 
aged and D-sorbitol treated MFC motor neuron cultures showed compensation of the observed distal differences of mitochondrial size between wildtype and TDP43 mutants and displayed a lysosomal size increase in TDP43 mutants. (D, H) Proximal and distal shape analysis of aged mitochondria (D) under D-sorbitol treatment showed a mimic of the proximal mitochondrial elongation of TDP43 mutants in the wildtype. For lysosomes $(\mathrm{H})$ the proximal phenotype got reversed and the TDP43 mutants were strongly influenced at the distal axon site. (E, I) Proximal and distal motility of mitochondria (E) and lysosomes (I) was rescued to normal speed in TDP43 mutants close to wildtype levels at both axon sites through D-sorbitol. (F, J) Dito for organelle displacement. (K, L) Representative immunofluorescence images of D-sorbitol-treated aging motor neurons negative for cytoplasmic TDP43 aggregation. (K) Wildtypes and TDP43 mutants showed no cytoplasmic TDP43 protein aggregation after treatment with $40 \mathrm{mM}$ D-sorbitol for 24h. (L) Increasing the D-sorbitol concentration to up to $400 \mathrm{mM}$ did not induce cytoplasmic TDP43 or FUS mislocalization/aggregation in wildtype motor neurons. Cell nuclei were stained with Hoechst. White arrowheads mark a single representative motor neuron. Scale bars: $25 \mu \mathrm{m}$. (B-J) Data of TDP43 mutants were pooled. Bar graphs (B) show mean with SEM. Box plots (CJ) show mean (+), median (line) and whiskers from 1-99 percentile (n=3-5, Kruskal-Wallis test with Dunn's post hoc test; asterisks compare cell lines (e.g. wt/TDP43 mutants) within a condition (e.g. distal), rhombs compare between conditions within a cell line; ${ }^{* \#} P \leq 0.05$, $\left.{ }^{* *} P \leq 0.01,{ }^{* * * \# \# \#} P \leq 0.001, * * * * \# \# \# \# \leq 0.0001\right)$. For detailed statistics see Suppl. Tables 2-9. 


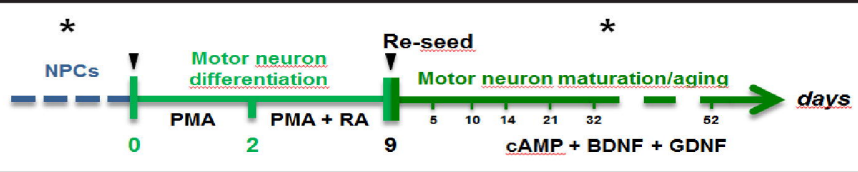

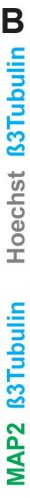

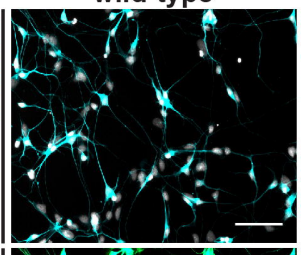

\section{TDP43 S393L}

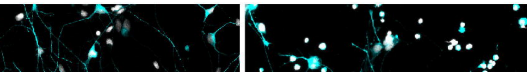

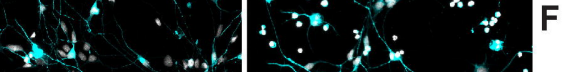
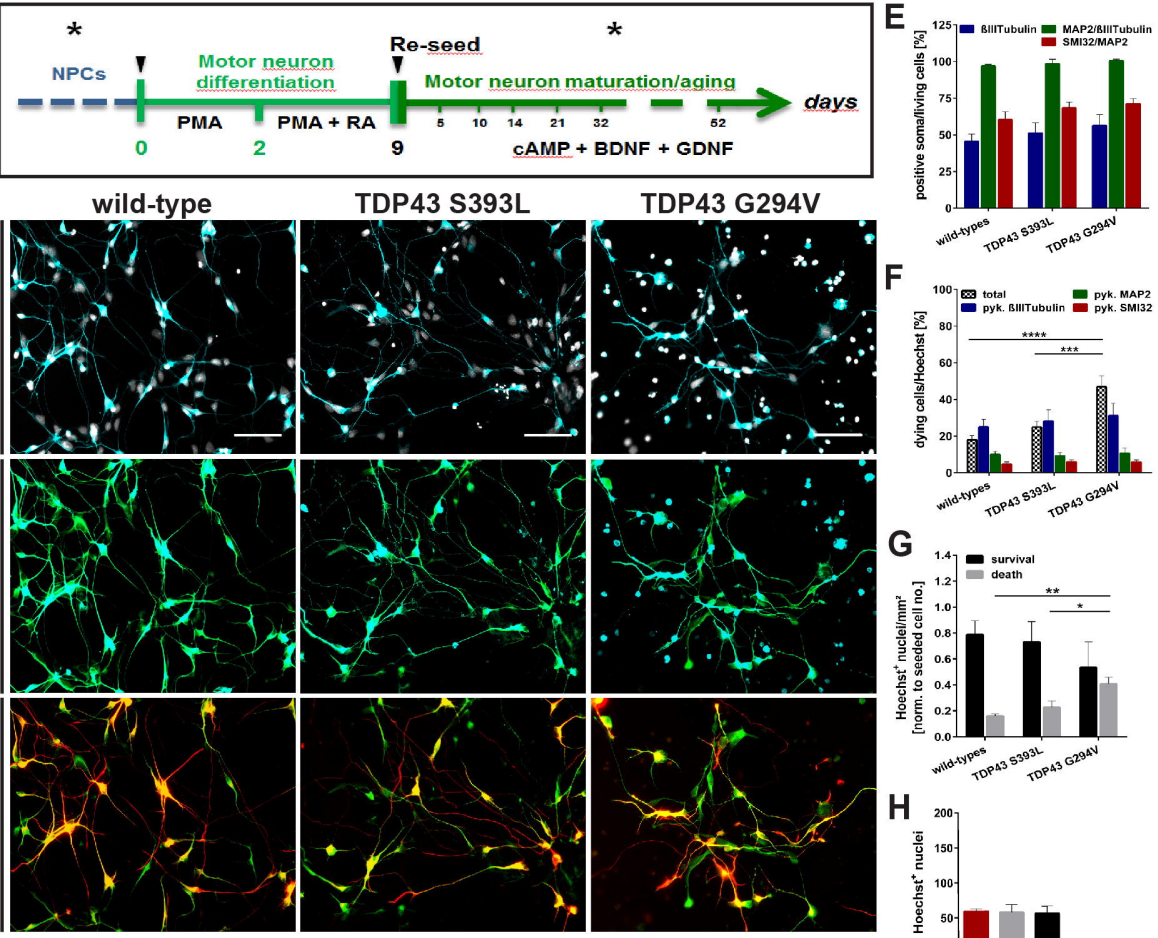

C

G
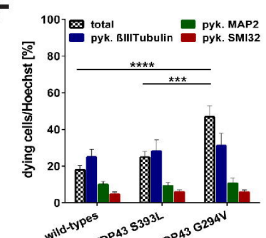

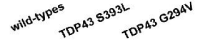

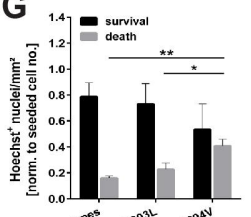

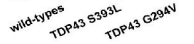

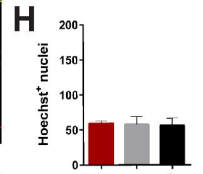

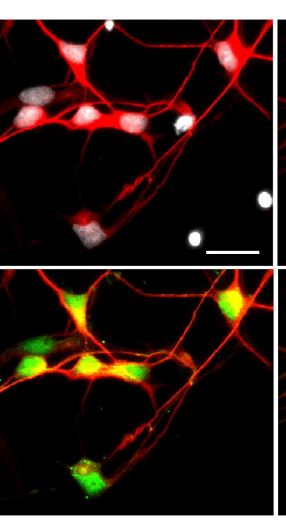
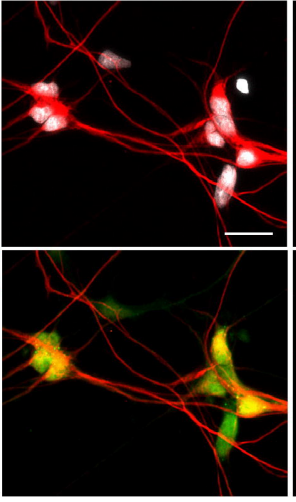

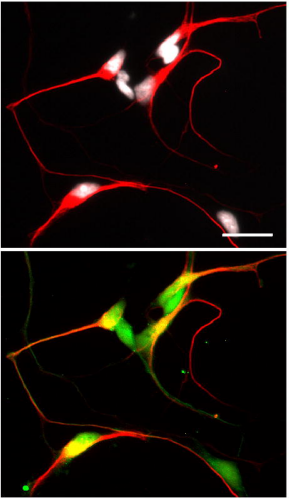

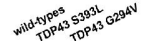

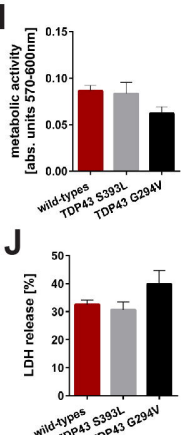

D

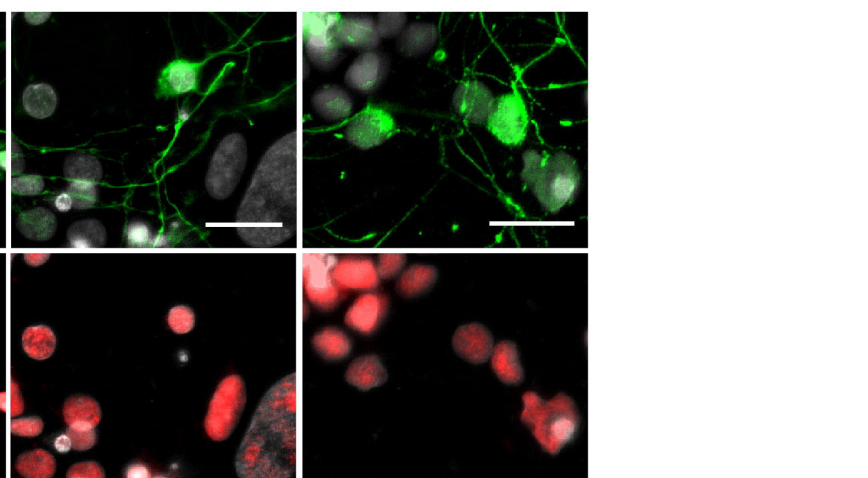



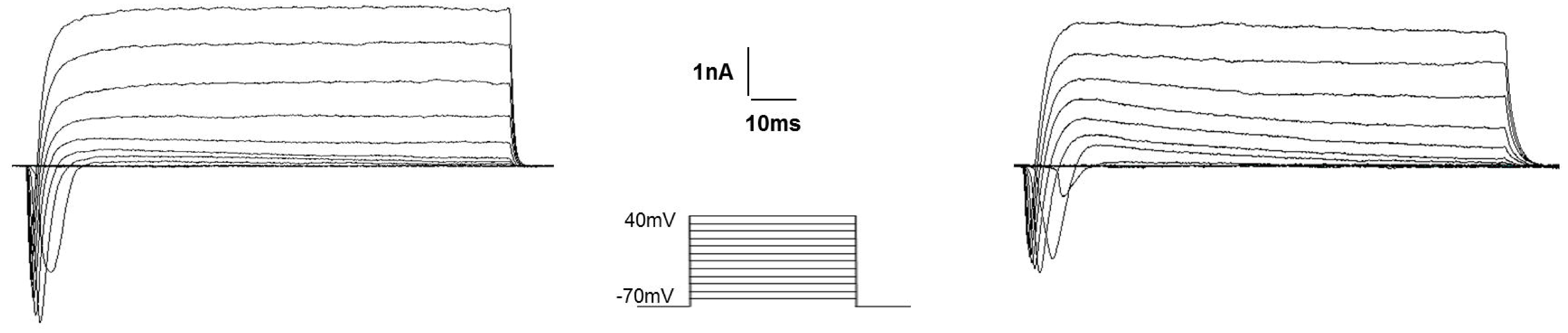

B

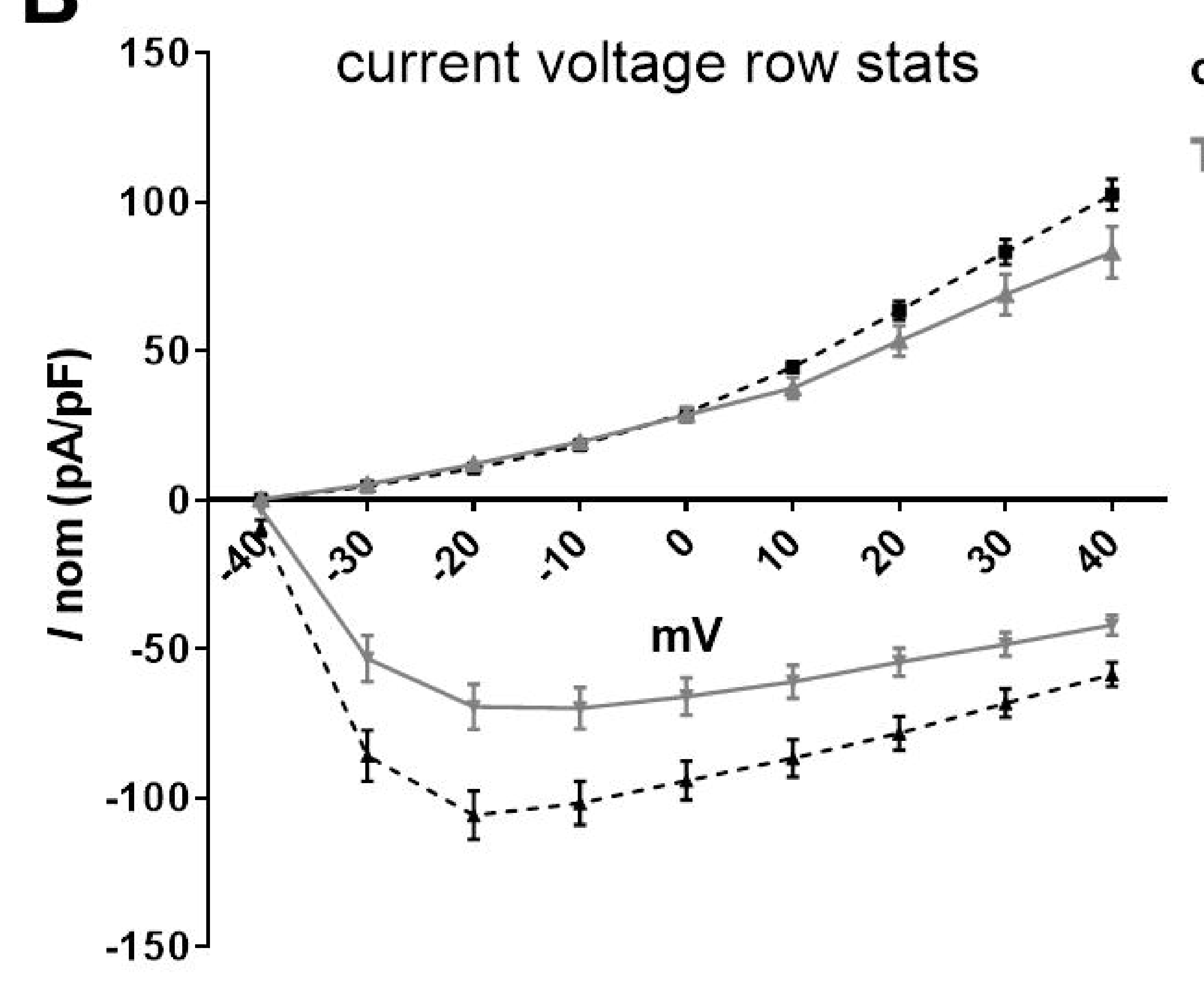

E
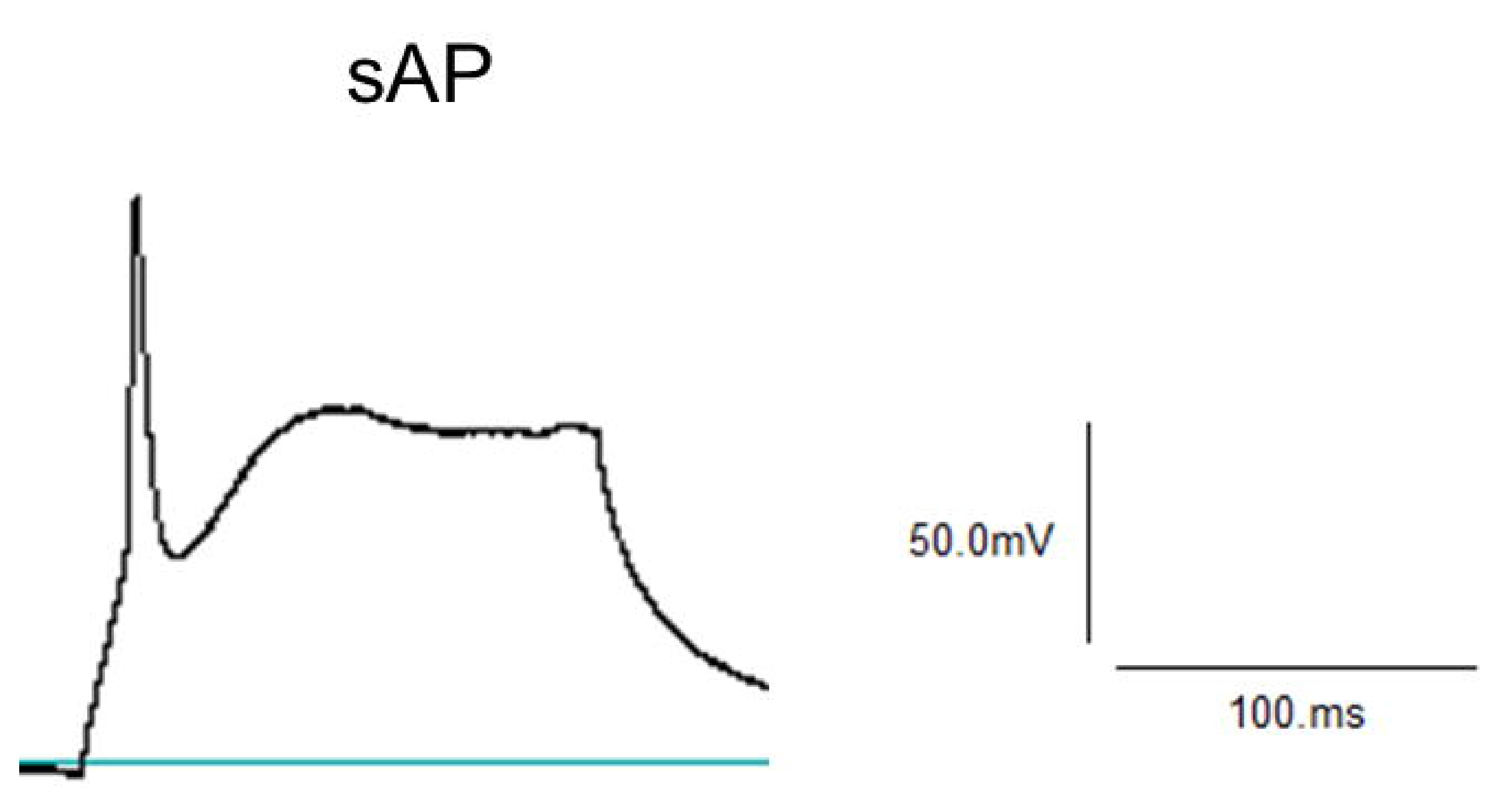

G spontaneous PSCs

20pA |

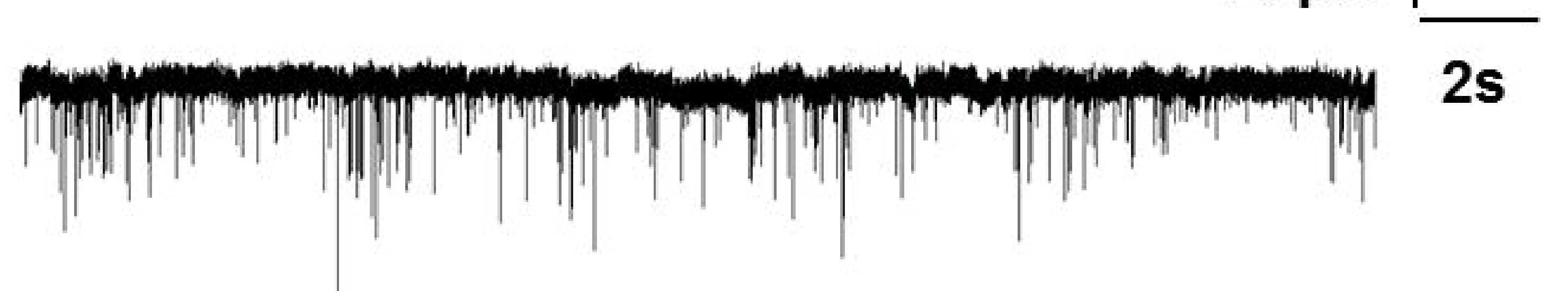

spontaneous APs

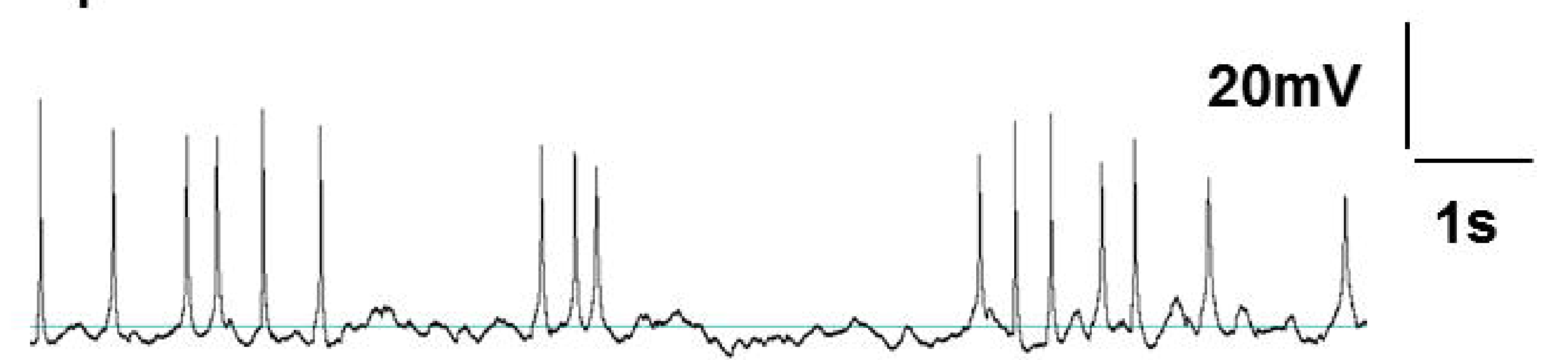

C
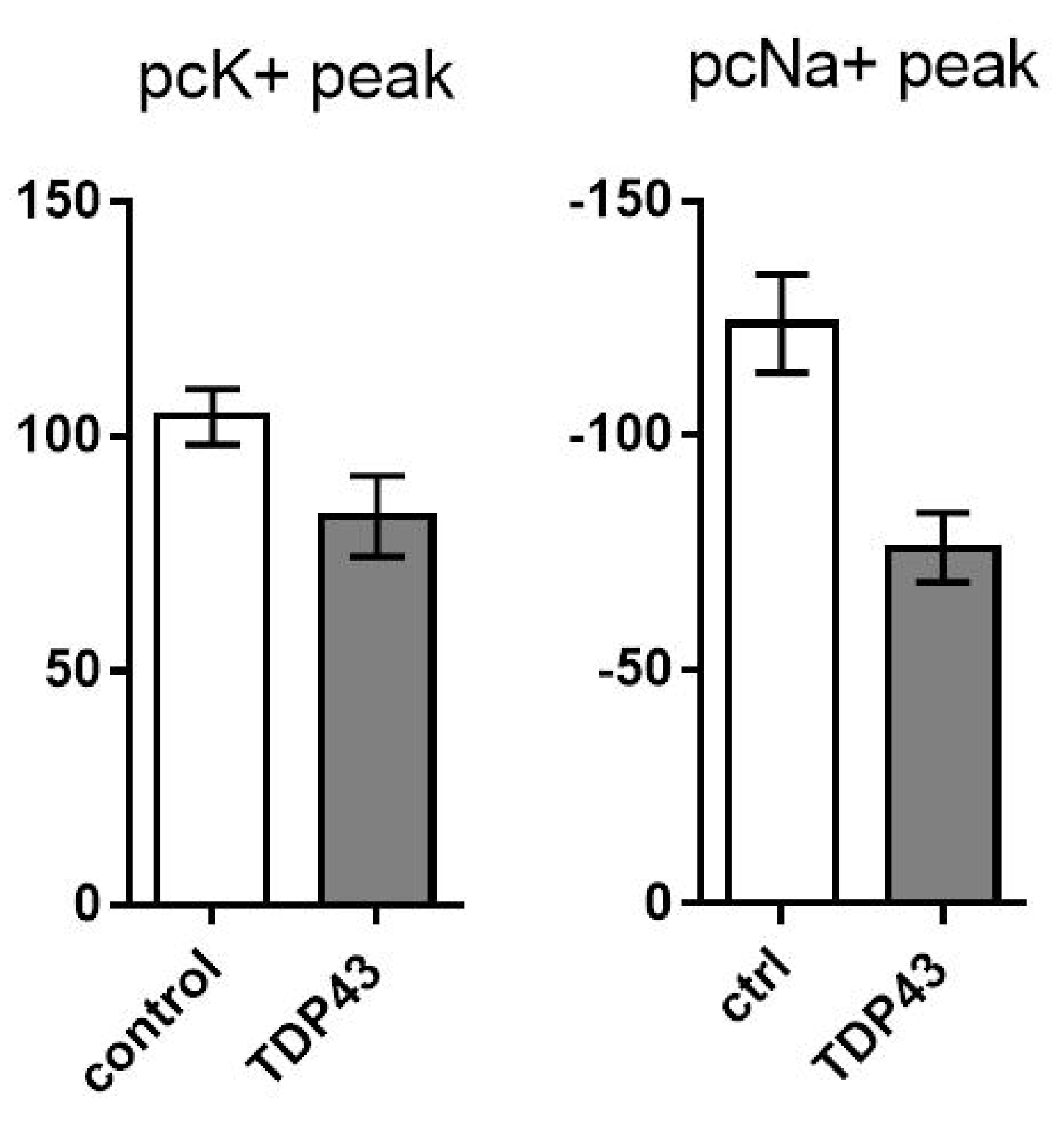

D

$\mathrm{Na}+/ \mathrm{K}+$

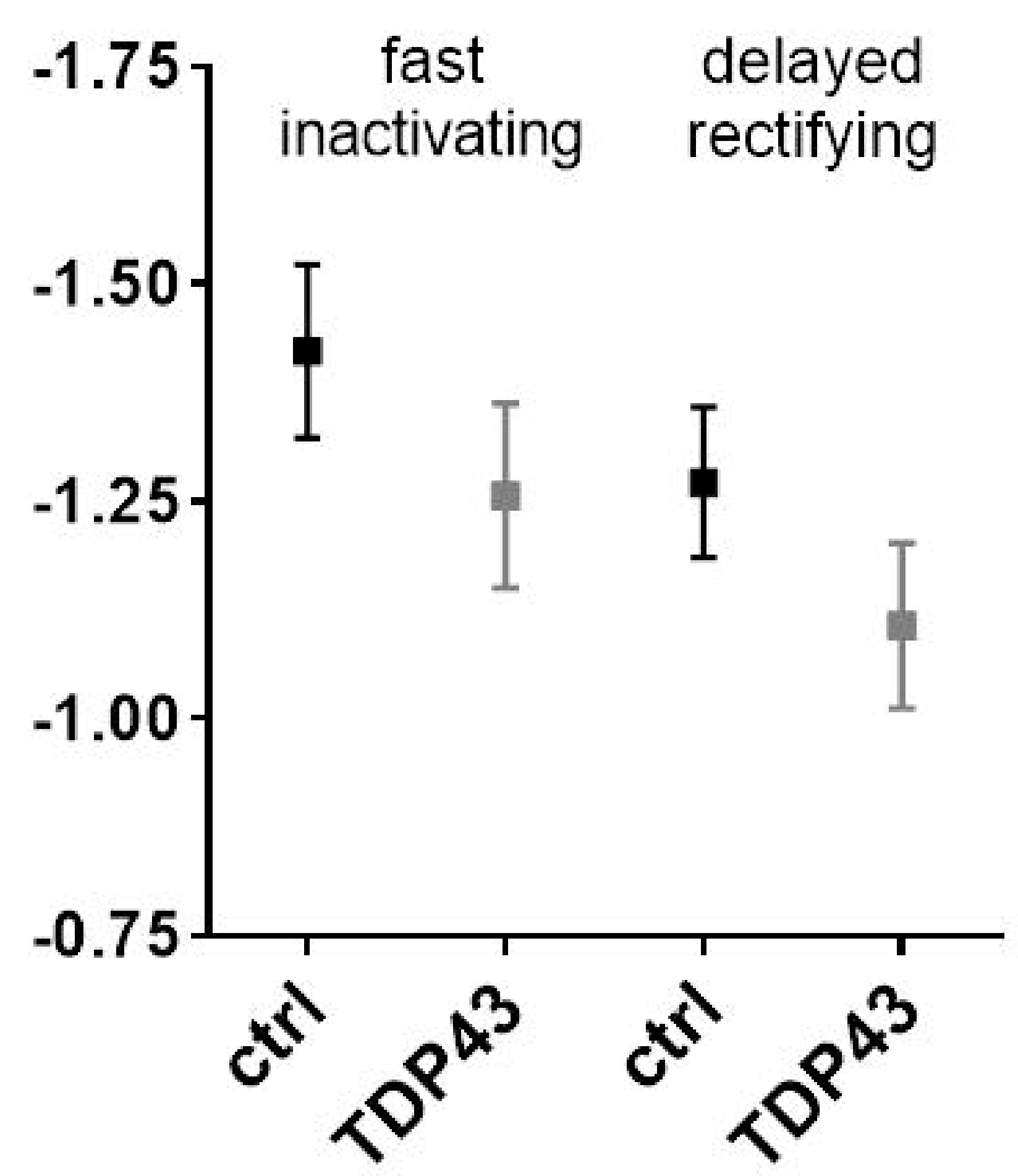

F

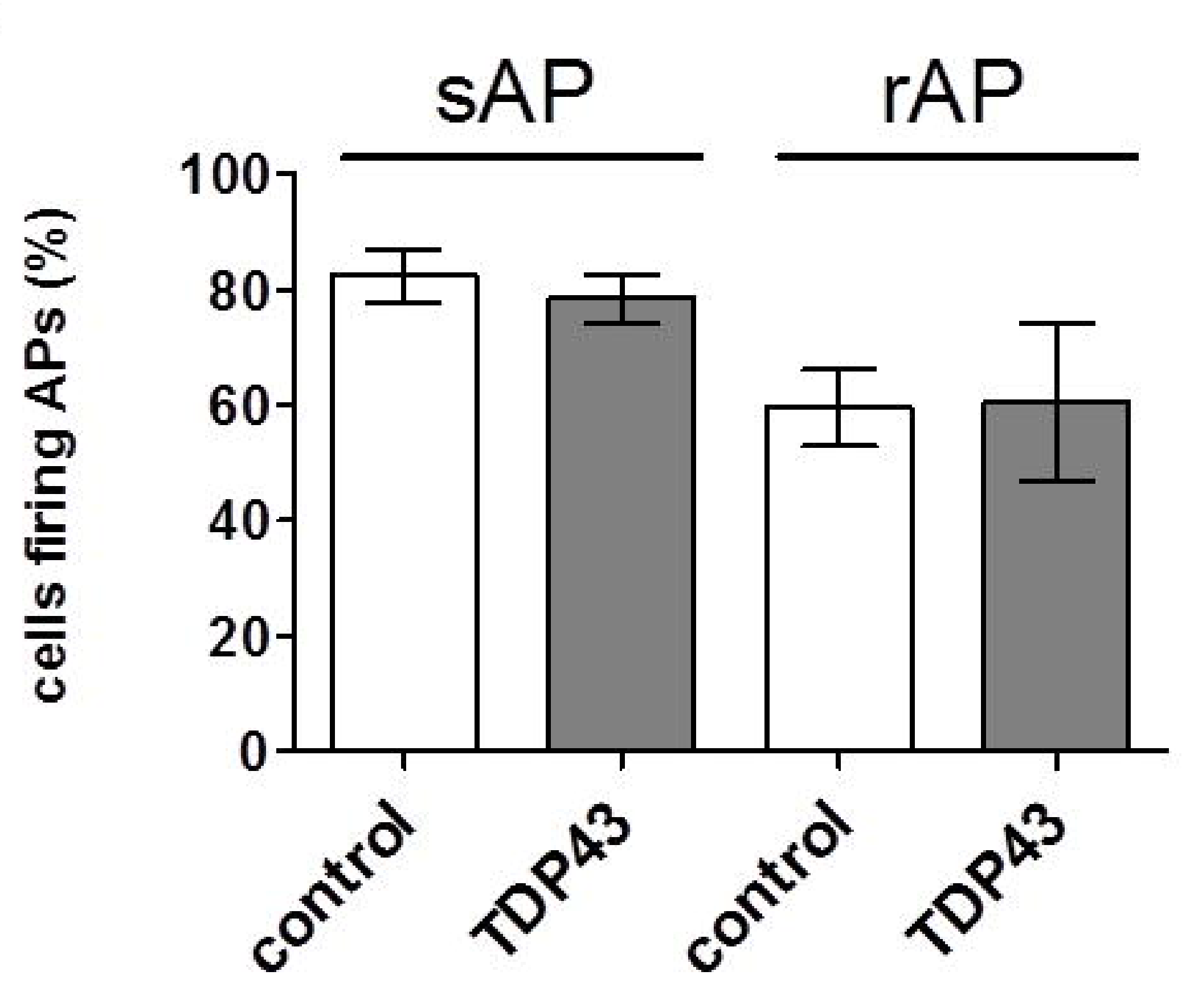

H

\section{Percentage}

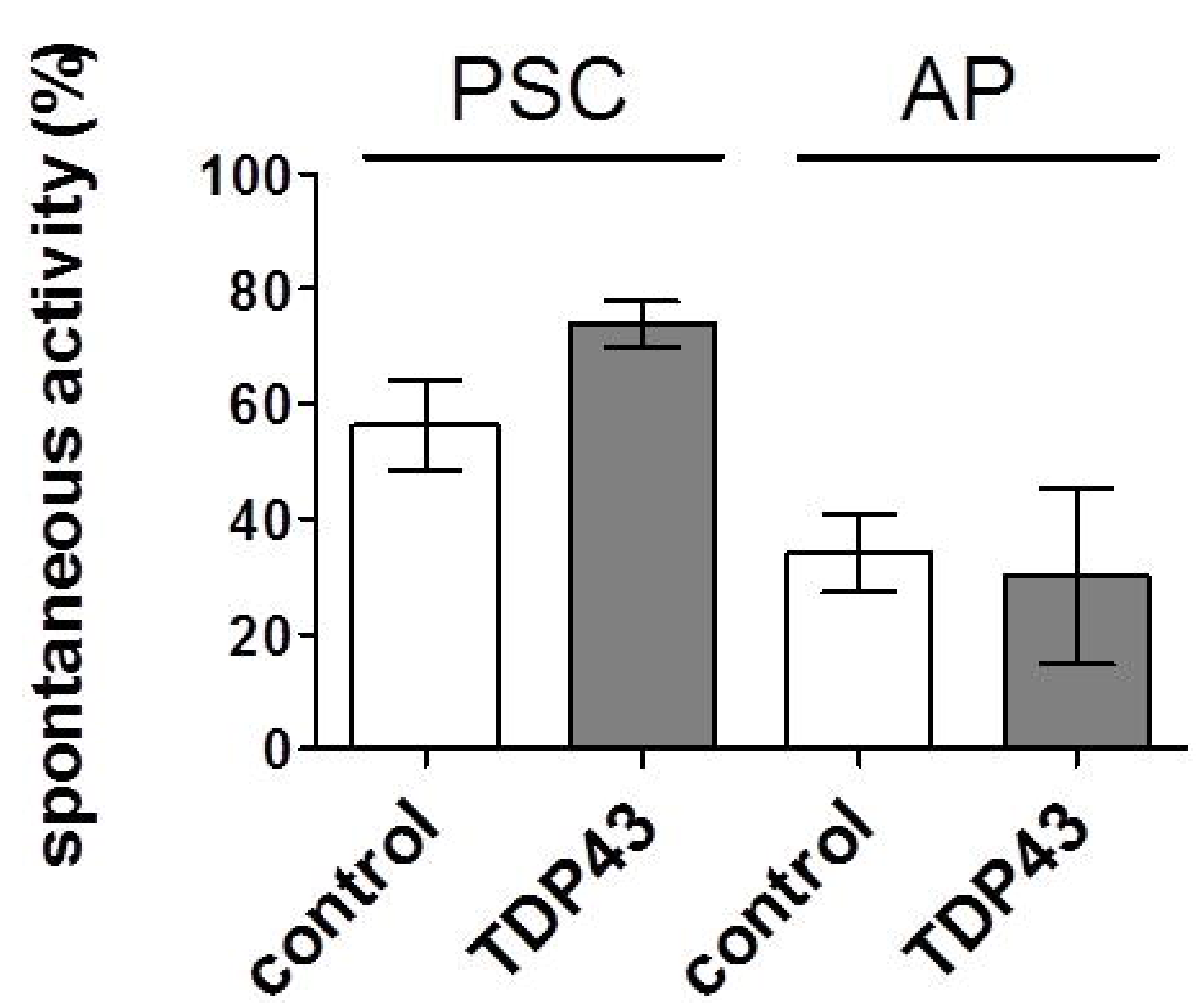

\section{Frequency}

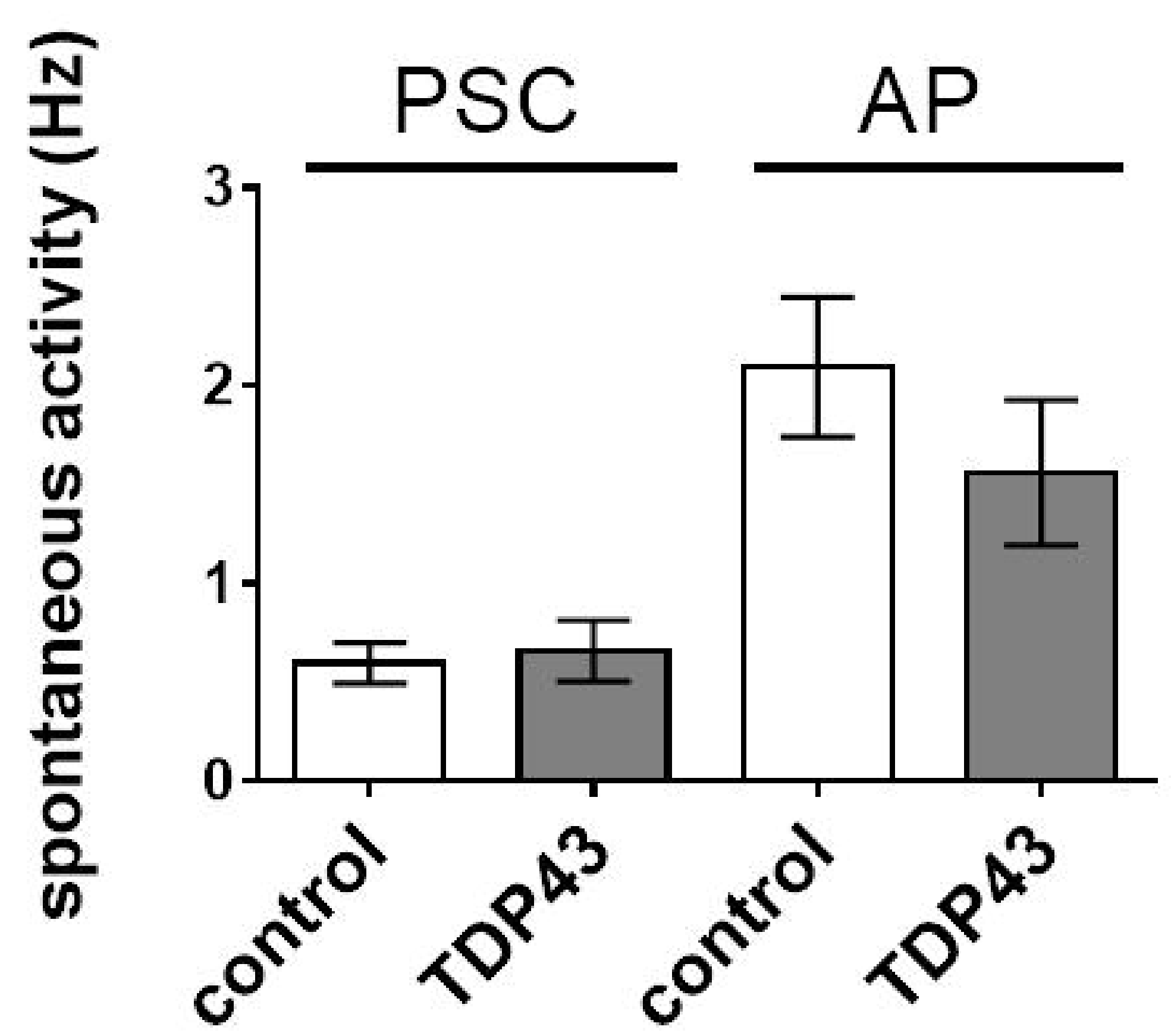


B

proximal

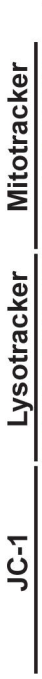

C

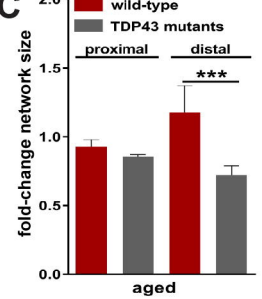

F

Mitotracker

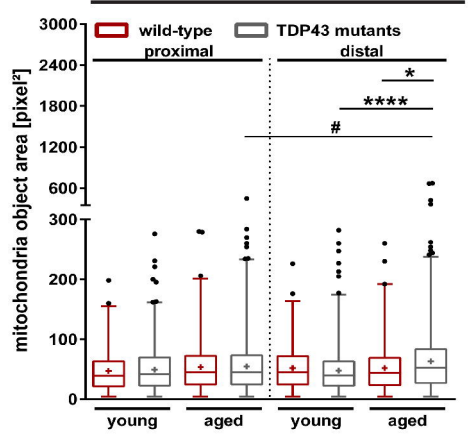

H

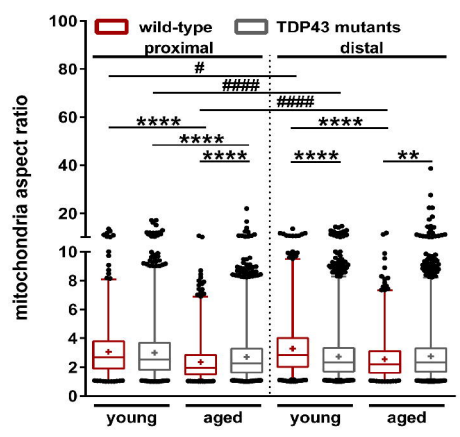

distal

young

aged
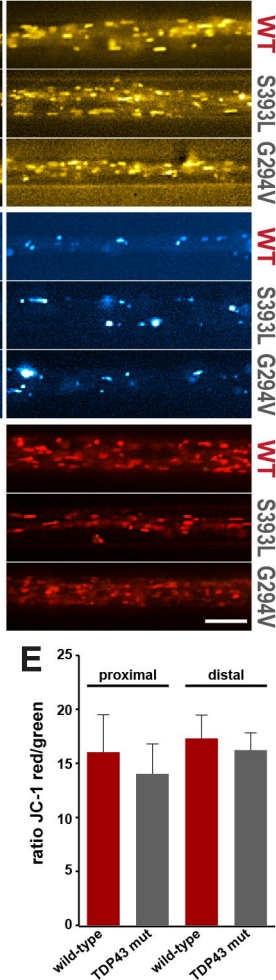

Lysotracker

G

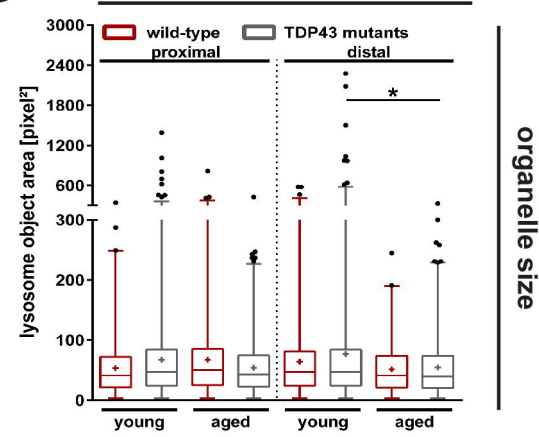

I

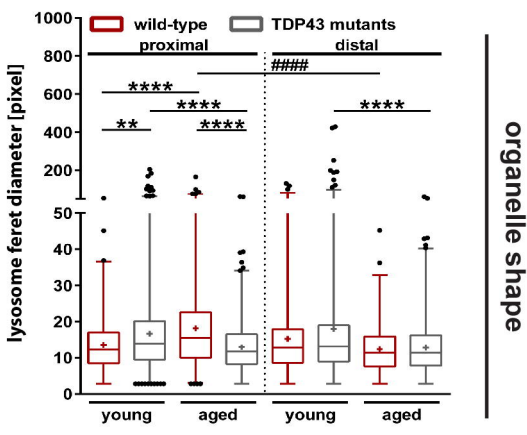




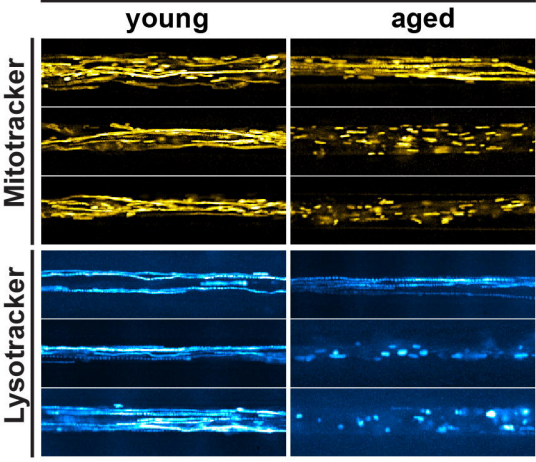

\section{Mitotracker}
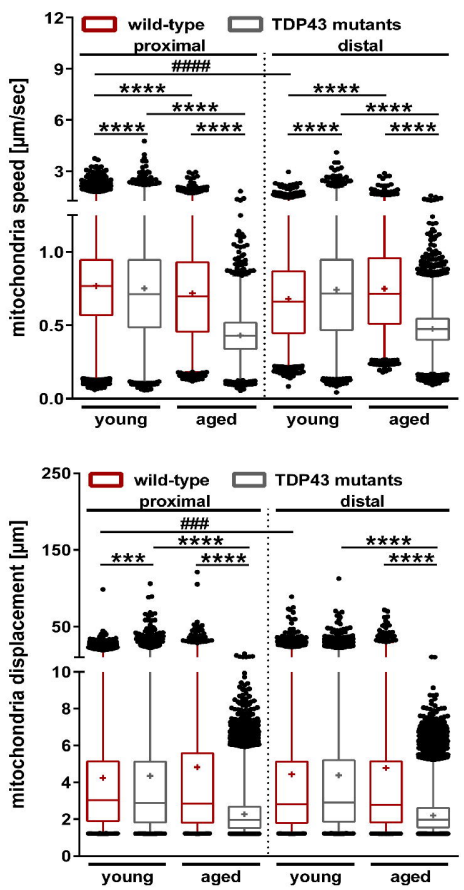

distal

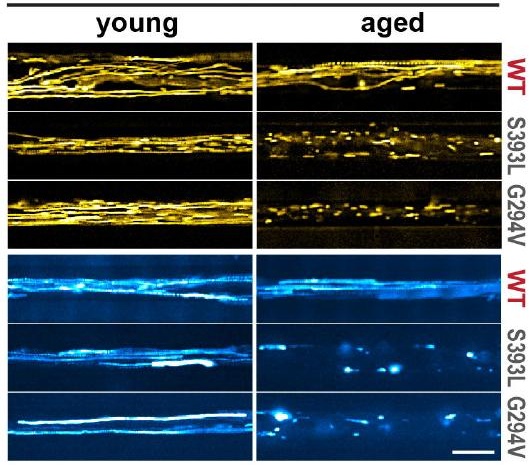

Lysotracker

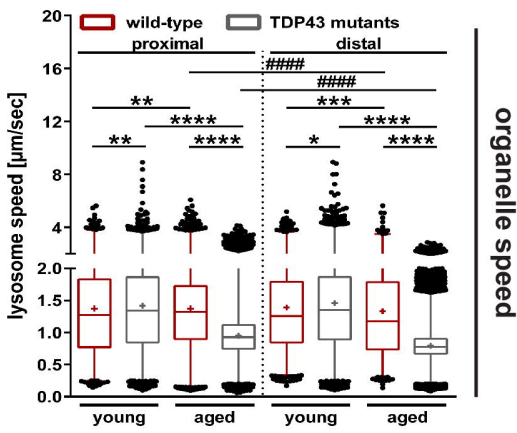

E

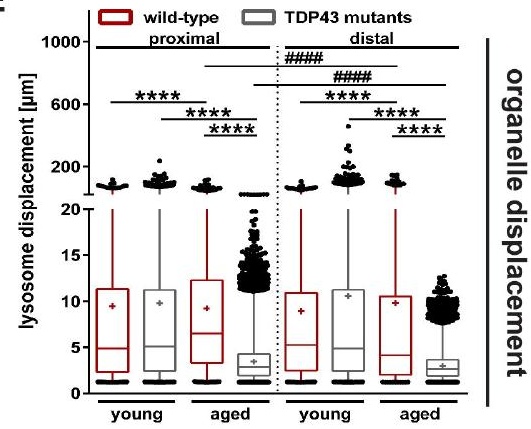




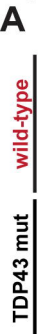

Mitotracker

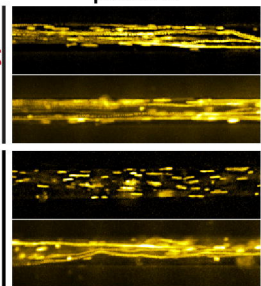

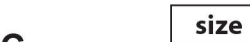

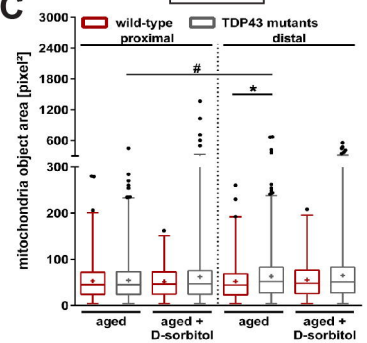

distal
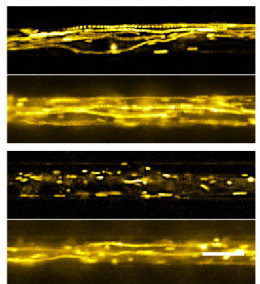

Lysotracker
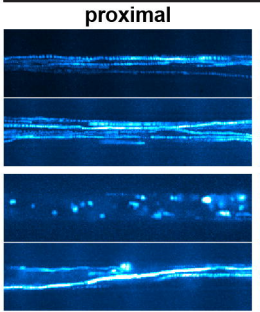
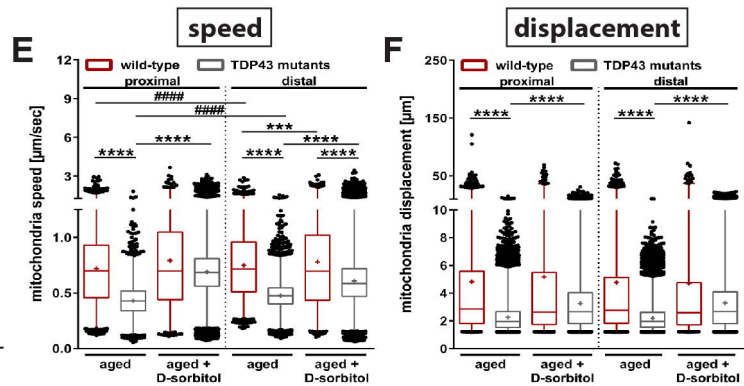

G
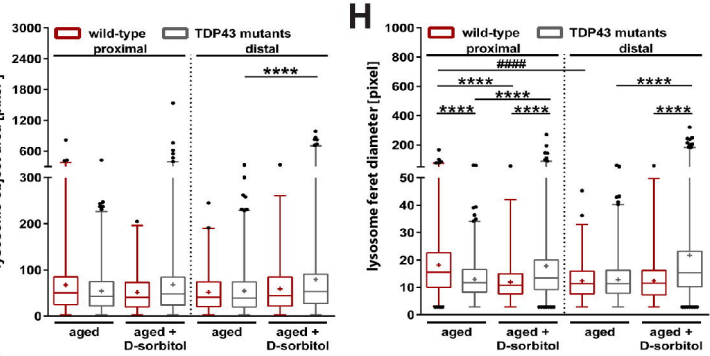

B

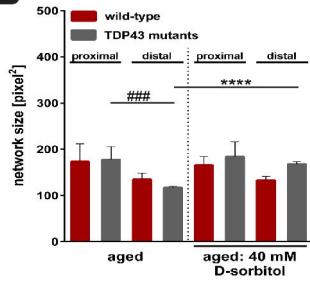

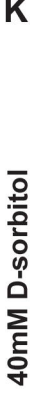

wild-type

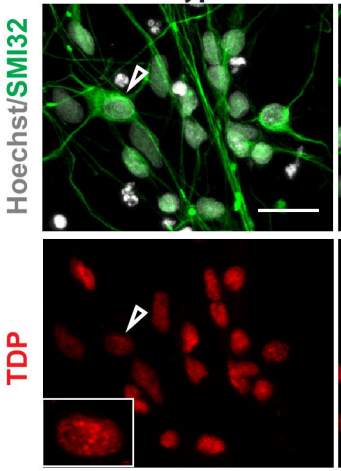

TDP43 S393L
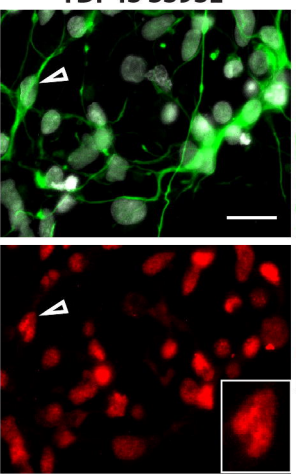

wild-type
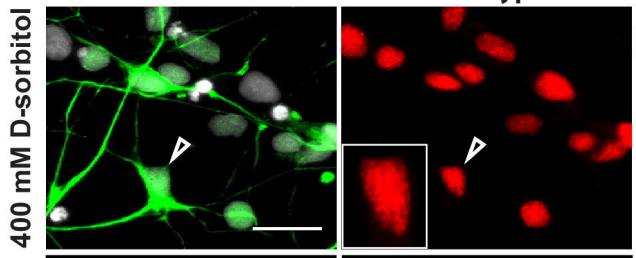

Hoechst/SMI32

\section{TDP43 G294V}
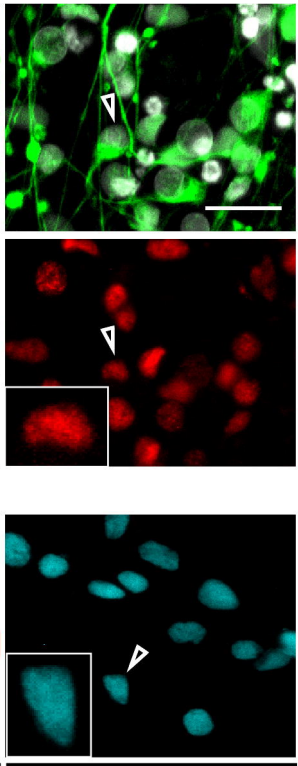

FUS

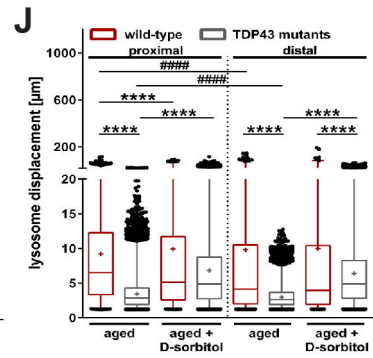

